ఠ

\title{
Plaque-hyaluronidase-responsive high-density- lipoprotein-mimetic nanoparticles for multistage intimal-macrophage-targeted drug delivery and enhanced anti-atherosclerotic therapy
}

\author{
This article was published in the following Dove Press journal: \\ International Journal of Nanomedicine \\ 13 January 2017 \\ Number of times this article has been viewed
}

\section{Mengyuan Zhang \\ Jianhua $\mathrm{He}$ \\ Cuiping Jiang \\ Wenli Zhang \\ Yun Yang \\ Zhiyu Wang \\ Jianping Liu}

Department of Pharmaceutics, China Pharmaceutical University, Nanjing,

People's Republic of China
Correspondence: Jianping Liu Department of Pharmaceutics, China Pharmaceutical University, 24 Tongjiaxiang, Nanjing 210009 , People's Republic of China

Tel +86258327 I293

Email jianpingliu1293@I63.com
Abstract: Increasing evidence has highlighted the pivotal role that intimal macrophage (iMФ) plays in the pathophysiology of atherosclerotic plaques, which represents an attractive target for atherosclerosis treatment. In this work, to address the insufficient specificity of conventional reconstituted high-density lipoprotein (rHDL) for iMФ and its limited cholesterol efflux ability, we designed a hyaluronan (HA)-anchored core-shell rHDL. This nanoparticle achieved efficient iMФ-targeted drug delivery via a multistage-targeting approach, and excellent cellular cholesterol removal. It contained a biodegradable poly (lactic-co-glycolic acid) (PLGA) core within a lipid bilayer, and apolipoprotein A-I (apoA-I) absorbing on the lipid bilayer was covalently decorated with HA. The covalent HA coating with superior stability and greater shielding was favorable for not only minimizing the liver uptake but also facilitating the accumulation of nanoparticles at leaky endothelium overexpressing CD44 receptors in atherosclerotic plaques. The ultimate iMФ homing was achieved via apoA-I after HA coating degraded by hyaluronidase (HAase) (abundant in atherosclerotic plaque). The multistage-targeting mechanism was revealed on the established injured endothelium-macrophage co-culture dynamic system. Upon treatment with HAase in vitro, the nanoparticle HA-(C)-PLGA-rHDL exhibited a greater cholesterol efflux capacity compared with conventional rHDL (2.43-fold). Better targeting efficiency toward iM $\Phi$ and attenuated liver accumulation were further proved by results from ex vivo imaging and $\mathrm{iM} \Phi$-specific fluorescence localization. Ultimately, HA-(C)-PLGA-rHDL loaded with simvastatin realized the most potent anti-atherogenic efficacies in model animals over other preparations. Thus, the HAase-responsive HDL-mimetic nanoparticle was shown in this study to be a promising nanocarrier for anti-atherogenic therapy, in the light of efficient iM $\Phi$-targeted drug delivery and excellent function of mediating cellular cholesterol efflux.

Keywords: covalent HA coating, rHDL, HAase response, multistage targeting for intimal macrophage, cholesterol efflux, anti-atherogenic efficacies

\section{Introduction}

Atherosclerosis has been widely recognized as a chronic inflammatory disease characterized by the build-up of fatty plaques within the arterial wall. Its associated acute coronary events rank among the leading causes of disability and death worldwide. ${ }^{1}$ Intimal macrophages (iMФs), the signature immune cells of atherosclerotic lesions, are pivotal contributors involved in the atherogenic process. These cells appear to participate in all stages in evolving atherosclerotic plaques as modulators of aberrant lipid metabolism and inflammatory responses. ${ }^{2-4}$ Abnormalities in cholesterol 
accumulation by iM $\Phi$ uptake of oxidized lipids and the subsequent formation of foam cells are believed to initiate the pathological cascade of atherosclerosis. The multiple obligatory atherogenic inflammations are promoted by iM $\Phi$ through secretion of various pro-inflammatory and cytotoxic factors, eventually causing the severity and destabilization of atherosclerotic plaques. Therefore, iM $\Phi$ has been actively pursued as a promising target for both diagnosis and treatment of atherosclerosis., ${ }^{5,6}$

Considering that high-density lipoprotein (HDL) belongs to a versatile class of natural nanoparticulates that possess distinctive reverse cholesterol transport (RCT) function (a process mediating the cellular cholesterol efflux), a favorable structure for incorporating cargoes, and selectively receptor (scavenger receptor-BI, SR-BI)-mediated endocytosis, utilizing reconstituted HDL (rHDL)-based biomimetic nanoplatform to deliver therapeutic agents to iM $\Phi$ and exert greatly atheroprotective actions would be a highly desirable treatment protocol. ${ }^{7-9}$ However, previous studies have demonstrated that although rHDL loaded with cardiovascular drug is able to deliver agents to $\mathrm{iM} \Phi$, there is an undesired, enormous liver accumulation of rHDL. This is due to the fact that the SR-BI is abundant not only in the $\mathrm{iM} \Phi$ of atherosclerotic lesions but also in the hepatocytes. ${ }^{10-12}$ To tackle the limited specificity of rHDL for iMФ, electrostatic adsorption of hyaluronan (HA) on the surface of spherical rHDL (s-rHDL) had been further applied to decrease the undesired uptake by liver and improve the plaque-targeting efficiency of s-rHDL in our previous work, attributing to the weak affinity of HA for hepatic receptors, but high affinity for CD44 receptors overexpressed in the impaired endothelium of atherosclerotic plaque. ${ }^{13-15}$

Nevertheless, this attempt might still be regarded as a limited success because of the following two drawbacks. On one hand, since s-rHDL consists of a lipid ester core (triglycerides and cholesteryl esters) covered with a phospholipid monolayer, its RCT function that accelerates lipid removal from iMФ would be disappointing, due to its high cholesterol concentration of lipid core and insufficient space of phospholipid monolayer for accepting peripheral cholesterol. ${ }^{16}$ And the import of exogenous cholesterol is also inappropriate for treating this hyperlipidemia-induced disease. Indeed, HDL exists in two distinct structural forms termed as s-HDL and discoidal HDL (d-HDL). During the RCT process, nascent d-HDL with a phospholipid bilayer obtains excess cholesterol from peripheral tissues and transforms into mature s-HDL. ${ }^{8}$ Compared with the phospholipid monolayer of s-rHDL, the phospholipid bilayer of d-rHDL serves as a higher-capacity acceptor for peripheral cholesterol in RCT and would be conducive to the fusion between d-rHDL and cell membranes, whereas drug leakage from d-rHDL occurs during the described remodeling behavior in the circulation. ${ }^{17-20} \mathrm{It}$ is thus ideal if HDL-mimetic nanoparticles could be fabricated through combining the advantages of d-HDL and s-HDL without inheriting their weaknesses. On the other hand, it is well known that SR-BI receptors are specifically recognized by the apolipoprotein A-I (apoA-I) on the HDL surface. ${ }^{11}$ Binding the anionic HA chains with rHDL only via electrostatic interaction is far from completely masking the negatively charged apoA-I due to the like-charge repulsion, and the unsheltered apoA-I still causes a non-ignorable amount of uptake by liver. ${ }^{13}$ Moreover, the physical HA coating might be abscised easily under the force of disturbed flow (d-flow) in the athero-prone area. ${ }^{21}$

Recent studies revealed that HDL-mediated cholesterol efflux in RCT primarily depends on the interaction between apoA-I and SR-BI or other efflux membrane proteins, but not on the core type of rHDL. ${ }^{22}$ As a biodegradable polymer that has been exploited widely in developing nanoparticles approved by the US Food and Drug Administration, poly (lactic-co-glycolic acid) (PLGA) may likely be a favored alternative to the lipid core for HDL-based nanoplatform. ${ }^{23-25}$ Thus, we anticipated that a core-shell rHDL (PLGA-rHDL) composed of a PLGA core functionalized with a phospholipid bilayer would be of enormous potential benefits in treating atherosclerosis: (i) PLGA core is capable of not only encapsulating high amounts of cardiovascular drugs without additional exogenous cholesterol but also maintaining a stable spherical structure of PLGA-rHDL; (ii) the slow-release property of PLGA polymer would be extremely favorable for the chronic disease treatment; and (iii) the phospholipid bilayer of core-shell rHDL would reinforce the fusion between nanoparticle and cell membranes as well as provide a larger space for receiving more cholesterol, thereby promoting cholesterol removal from iM $\Phi$.

Meanwhile, it is worth noting that different routes for the attachment of HA to rHDL may generate different targeting features of nanocarriers, especially the ability to tackle the hemodynamic drag and unwanted off-targeting. Considering that the carboxylic acid residues of HA can also be offered as functional groups for covalent binding, decoration of HA on rHDL via covalent attachment seems preferable rather than electrostatic adsorption owing to the superior stability of chemical bonds. Most importantly, amino groups in apoA-I could also serve as anchors for HA chains, which would achieve better HA-binding capacity and more effective 
shielding for evading the liver recognition via apoA-I before reaching the atherosclerotic lesions. ${ }^{20,26}$ Therefore, covalently attaching HA to the surface of PLGA-rHDL (HA-(C)-PLGArHDL) was proposed in this study, in order to achieve more efficient iM $\Phi$-targeted delivery.

What is more, the underlying mechanisms by which HA-coated rHDL entered into atherosclerotic lesions and accessed into iM $\Phi$ were not illuminated well in our previous research. In view of the increased permeability of impaired endothelium and wide availability of hyaluronidase (HAase) in extracellular matrix of plaque, ${ }^{27}$ we hypothesized that HA-(C)-PLGA-rHDL would achieve efficient iMФ-targeted delivery via an ingenious multistage-targeting process (Figure 1). At the initial stage, in virtue of the covalent HA coating with superior stability and greater shielding for evading the recognition of liver, HA-(C)-PLGA-rHDL not only reduces the unwanted liver accumulation but also preferentially accumulates and prolongs its retention in the injured endothelium of plaque via active CD44-mediated targeting. The capping HA layer would be degraded by HAase within plaque at the subsequent stage, allowing the exposure of PLGA-rHDL as while the protection by HA coating switches off, iMФ-targeting ability of apoA-I via SR-BI-mediated endocytosis and cholesterol removal turns on. A further point of interest of this study was to elucidate the potential targeting mechanisms of HA-(C)-PLGA-rHDL in an established injured endothelium-macrophage cell co-culture dynamic system that resembles the in vivo impaired vascular endothelial barrier of atherosclerotic plaque, which would make us better understand this HAase-responsive drug delivery system that exerts the specific targetability for iMФ. Furthermore, to validate whether HA modification through covalent bonding could significantly enhance iM $\Phi$-targeting ability and atheroprotective functions compared with that of electrostatic adsorption under the d-flow conditions, HA electrostatically attached to rHDL (HA-(E)-PLGA-rHDL) was also investigated in the present study.

Taken together, the principal aim of this study was to design a high-performance HDL-mimetic nanovehicle, in order to decrease the undesired liver uptake, enlarge the
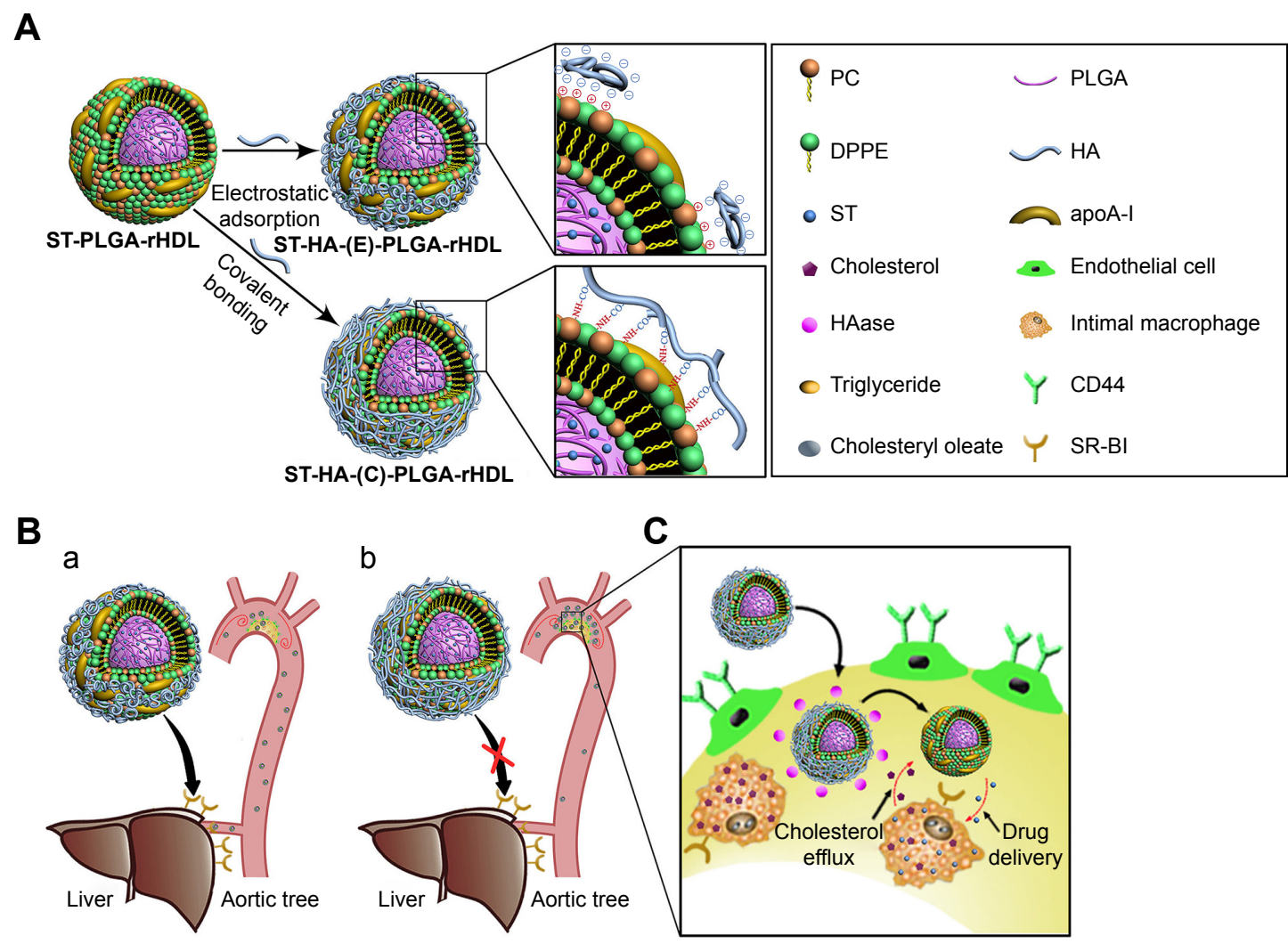

Figure I Schematic depiction (A) of the composition of ST-PLGA-rHDL, ST-HA-(E)-PLGA-rHDL, and ST-HA-(C)-PLGA-rHDL. Overview (B) of the higher iMФ-targeting efficiency and reduced liver accumulation of HA-(C)-PLGA-rHDL (b) than that of HA-(E)-PLGA-rHDL (a) due to the better shielding effect of covalent HA coating than electrostatic HA binding. Overview (C) of multistage-targeting process of HA-(C)-PLGA-rHDL through firstly accumulating in the injured endothelium of plaque via active CD44-mediated targeting, and then delivering agents into iM $\Phi$ via SR-BI receptors as well as promoting cellular cholesterol efflux.

Abbreviations: ST, simvastatin; PLGA, poly (lactic-co-glycolic acid); rHDL, reconstituted high-density lipoprotein; HA, hyaluronan; (E), electrostatically attached; (C), covalently attached; iMФ, intimal macrophage; SR-BI, scavenger receptor-BI; PC, egg phospholipid; DPPE, I,2-dipalmitoyl-sn-glycero-3-phosphoethanolamine; apoA-I, apolipoprotein A-I; HAase, hyaluronidase. 
drug accumulation in iM $\Phi$ of atherosclerotic lesion, as well as maintain the attractive ability of promoting cholesterol removal from iM $\Phi$. To fulfill this goal, an HAase-responsive core-shell rHDL covalently bonded with HA (HA-(C)PLGA-rHDL) was synthesized by covalent linkage of the carboxyl groups of targeting ligand HA to the amino groups of 1,2-dipalmitoyl-sn-glycero-3-phosphoethanolamine (DPPE) and apoA-I of PLGA-rHDL. Simvastatin (ST) was selected as the model drug. In addition to its lipid-lowering effect on hepatocytes, various research has established the potent immunomodulating effects of ST in macrophage including inhibiting the expression of matrix metalloproteinase (MMP), and reducing macrophage infiltration and other pro-inflammatory responses, which are greatly hindered by the poor systemic bioavailability and adverse effects of ST after being administrated orally for a long time. ${ }^{28-31}$ Thus, ST was loaded in to HA-(C)-PLGA-rHDL (ST-HA-(C)-PLGArHDL) focusing on its intriguing pleiotropic effects. In vitro characterizations including physicochemical properties, morphology, and drug release of ST-HA-(C)-PLGA-rHDL were systematically determined. The $\mathrm{i} \Phi \Phi$-targeting abilities and mechanisms were then explored using established injured endothelium-macrophage cell co-culture dynamic system. Moreover, the bioactivity of mediating cellular efflux cholesterol was assessed in the murine macrophage cell line. For in vivo studies, atherosclerotic iM $\Phi$-targeting property and anti-atherogenic efficacies were elaborately examined in atherosclerotic rabbit models.

\section{Materials and methods Materials}

ST was kindly donated by Shangyu Jing Xin Pharmacy Company (Shaoxing, People's Republic of China). PLGA (lactide:glycolide 50:50, inherent viscosity $0.2 \mathrm{dL} / \mathrm{g}$, molecular weight [MW] $15 \mathrm{kDa}$ ) was purchased from Shandong Medical Device Research Institute (Jinan, People's Republic of China). D-alpha-Tocopheryl polyethylene glycol 1000 succinate (TPGS) and octadecylamine (OL) were obtained from Sigma-Aldrich Chemie GmbH (Munich, Germany). Egg phospholipid (PC; Lipoid S100) was obtained from Lipoid $\mathrm{GmbH}$ (Ludwigshafen, Germany). DPPE was purchased from Shanghai Advanced Vehicle Technology Pharmaceutical Ltd. Co. 1-Ethyl-3-(3-dimethylaminopropyl) carbodiimide (EDC) and N-hydroxy-succinimide (NHS) were obtained from Aladdin Reagent Database Inc. (Shanghai, People's Republic of China). Sodium hyaluronic acid (HA, 200-400 kDa) was purchased from Freda Biochem Co., Ltd. (Jinan, People's Republic of China). ApoA-I was isolated from the albumin by-product as depicted previously. Block lipid transport-1
(BLT-1) was purchased from ChemBridge (San Diego, CA, USA). 6-[(7-nitro-2,1,3-benzoxadial-4-yl)amino]-cholest-5en-3-ol (NBD-cholesterol) was from Cayman Chemical (Ann Arbor, MI, USA). Bovine serum albumin was purchased from Sunshine Biotech Co., Ltd. (Shanghai, People's Republic of China). ST capsule was bought from Xiamen Fu Man Pharmaceutical Co., Ltd. (Xiamen, People's Republic of China). Fluorescent carbocyanine dyes, 1,1'-dioctadecyl3,3,3',3'-tetramethylindodicarbocyanine perchlorate (DiD; ex $644 \mathrm{~nm} / \mathrm{em} 663 \mathrm{~nm}$ ) and 1,1'-dioctadecyl-3,3,3',3'-tet ramethylindotricarbocyanine iodide (DiR; ex $748 \mathrm{~nm} / \mathrm{em}$ $780 \mathrm{~nm}$ ) were purchased from Fanbo Biochemicals Co., Ltd. (Beijing, People's Republic of China). All other chemicals and reagents were of analytical grade.

\section{Preparation of ST-loaded nanoparticles}

PLGA-lipid nanoparticle loaded with ST (ST-PLN) was prepared using a nanoprecipitation method with a modified protocol. ${ }^{32}$ Briefly, a mixture of lipid (DPPE:PC:OL mole ratio, $2: 1: 0.35)$ and $1.2 \mathrm{mg} / \mathrm{mL}$ of TPGS was dissolved in methanol/ chloroform $(1: 1, \mathrm{v} / \mathrm{v})$ and dried overnight in an egg-plant flask to form a dry film. Phosphate-buffered saline (PBS) buffer (0.01 M, pH 7.4) was then added to hydrate the dried film, and the obtained hybrid suspension was vortexed thoroughly followed by ultrasonication in an ice bath to obtain the vesicle solution. The oil phase was prepared by dissolving ST with $10 \mathrm{mg} / \mathrm{mL}$ of PLGA and $4.0 \mathrm{mg} / \mathrm{mL}$ of TPGS in acetonitrile, and injected into the preheated vesicle solution under magnetic stirring. After self-assembly with continuous stirring at $40^{\circ} \mathrm{C}$, nanoparticles were washed three times by ultracentrifugation (10 kDa cutoff, Amicon Ultra-15 filter, Millipore, Bedford, USA) and subsequently resuspended in PBS again.

Next, PLGA-rHDL loaded with ST (ST-PLGA-rHDL) was obtained by incubating ST-PLN with an equivalent volume of $\mathrm{pH}$ 7.4 PBS buffer containing $20 \mathrm{mg}$ of apoA-I and $35 \mathrm{mg}$ of sodium cholate under centrifugation at $600 \mathrm{rpm}$ at $4^{\circ} \mathrm{C}$ for $8 \mathrm{~h}$, and the dispersion was then dialyzed to remove excess sodium cholate..$^{10,13}$

Furthermore, HA-(C)-PLGA-rHDL loaded with ST (ST-HA-(C)-PLGA-rHDL) was synthesized following a modified method as described by Gan et al. ${ }^{33}$ Ten milligrams of HA was dissolved in $10 \mathrm{~mL}$ of water ( $\mathrm{pH} 4.0$ ) overnight to allow complete swelling, and preactivated by $1 \mathrm{M}$ equivalent of coupling agents EDC and NHS for $2 \mathrm{~h}$ at $37^{\circ} \mathrm{C}$. After the addition of activated HA to ST-PLGA-rHDL solution $(1: 2, \mathrm{v}: \mathrm{v})$, the reaction continued under stirring for $18 \mathrm{~h}$ at $25^{\circ} \mathrm{C}$, and the resulting ST-HA-(C)-PLGA-rHDL was separated from free $\mathrm{HA}$ and residual reagents by centrifugation $(13,000 \mathrm{rpm})$ for $30 \mathrm{~min}$ at $4^{\circ} \mathrm{C}$. 
Additionally, ST-loaded s-rHDL (ST-s-rHDL) and ST-HA(E)-PLGA-rHDL formed by the electrostatic absorption between HA and cationic ST-PLGA-rHDL were obtained according to the same methods reported in our previous study, respectively. ${ }^{10,13}$ PLGA nanoparticles loaded with ST (ST-PN) was also prepared according to a modified nanoprecipitation technique reported by Zhu et al. ${ }^{34}$

\section{In vitro characterizations}

Mean size, zeta potential, EE, and DL

Mean size and surface zeta potential of prepared nanoparticles were determined using dynamic light scattering (DLS) analyzer (Zetasizer $3000 \mathrm{HAS}$; Malvern Instruments, Malvern, UK). Each measurement of samples diluted appropriately was made in triplicate at $25^{\circ} \mathrm{C}$.

After removal of unentrapped drug through ultrafiltration method using Amicon Ultra-4 centrifugal filter tubes (10 kDa cutoff; Millipore), the entrapment efficiency (EE) and drugloading (DL) of various ST-loaded nanoparticles were calculated using the following equations, where $W_{0}$ and $W$ are the total amount of ST in each nanoparticle suspension and ultrafiltrate (free ST), respectively, and $Q_{0}$ is the total amount of the feeding materials:

$$
\begin{aligned}
& \operatorname{EE}(\%)=\frac{W_{0}-W}{W_{0}} \times 100 \% \\
& \operatorname{DL}(\%)=\frac{W_{0}-W}{Q_{0}} \times 100 \%
\end{aligned}
$$

The amount of ST was quantified by high-performance liquid chromatography (HPLC; Agilent 1200 series; Agilent Technologies, Palo Alto, CA, USA) equipped with a ultraviolet detector at $238 \mathrm{~nm}$ using a shim-pack VP-ODS column $(150 \times 4.6 \mathrm{~mm}, 5 \mu \mathrm{m})$ at $30^{\circ} \mathrm{C}$. The mobile phase consisted of acetonitrile and water $(80: 20, \mathrm{v} / \mathrm{v})$ with a flow rate of $1.0 \mathrm{~mL} / \mathrm{min}$.

\section{HA-binding efficiency}

ST-HA-(E)-PLGA-rHDL and ST-HA-(C)-PLGA-rHDL were separated from free HA by centrifugation for $30 \mathrm{~min}$ at $4^{\circ} \mathrm{C}$. The quantity of free $\mathrm{HA}$ in the supernatant fraction was determined by the hexadecyl trimethyl ammonium bromide (CTAB) turbidimetric method. ${ }^{33}$

Specifically, the supernatant containing free HA was incubated with $0.2 \mathrm{M}$ sodium acetate buffer at $37^{\circ} \mathrm{C}$ for 10 min in a 96-well plate. Ten millimolar CTAB solution was then added to the mixture, and subsequently, the absorbance of the mixture was measured at $570 \mathrm{~nm}$ within
10 min against the blank using a microplate reader (Varioskan Flash; Thermo Fisher Scientific, Waltham, MA, USA). The HA-binding efficiency $\left(B E_{\mathrm{HA}}\right)$ was calculated using the following formula, where $C$ and $C_{0}$ are the amount of free HA and the initial amount of HA added to the reaction, respectively:

$$
B E_{\mathrm{HA}}(\%)=\frac{C_{0}-C}{C_{0}} \times 100 \%
$$

\section{Morphology}

The morphology of various ST-loaded nanoparticles was visualized by transmission electron microscopy (TEM; H-7650; Hitachi High Technologies Corporation, Tokyo, Japan). One drop of each nanostructure after appropriate dilution was stained with $2 \%(\mathrm{w} / \mathrm{v})$ phosphotungstic acid, placed onto a copper grid with carbon film, air-dried at room temperature, and then characterized visually under the TEM.

\section{In vitro release in the HAase environment}

To measure the amount of release of ST from ST-PLN, ST-PLGA-rHDL, ST-HA-(E)-PLGA-rHDL, and ST-HA(C)-PLGA-rHDL in the presence of HAase, $3 \mathrm{~mL}$ of various nanoparticle dispersions containing $2 \mathrm{mg} / \mathrm{mL}$ HAase (Sigma-Aldrich Ltd., St Louis, MO, USA) were sealed in a dialysis bag (MW cutoff $=8-12 \mathrm{kDa}$ ), respectively. The dialysis bag was then immersed in $200 \mathrm{~mL}$ release buffer $(0.05 \%$ sodium dodecyl sulfate in PBS, $\mathrm{pH} 7.4)$ with gentle stirring at $37^{\circ} \mathrm{C}$. At designated time intervals, each $0.5 \mathrm{~mL}$ of release buffer was collected and compensated with the same volume of prewarmed fresh medium over $72 \mathrm{~h}$. The ST concentration of the collected release buffer at each time point was assayed by HPLC. The release profile of ST from each nanoparticle in the absence of HAase was also determined as a control.

\section{In vitro iМФ-targeting property Injured endothelium-macrophage co-culture system establishment}

Firstly, human umbilical vein endothelial cells (HUVECs, the kind gift from the Atherosclerosis Research Centre, Nanjing Medical University, Nanjing, People's Republic of China) were seeded onto gelatin-coated Transwell polycarbonate filters $(0.4 \mu \mathrm{m}$ pore, $6.5 \mathrm{~mm}$ membrane diameter; Corning Incorporated, Corning, NY, USA) and cultured for 3 days to form a restrictive endothelial monolayer. Then, RAW264.7 macrophage cell line (iM $\Phi$ ) in logarithmic growth phase was plated into the lower compartments of Transwell chambers and co-cultured with HUVECs on the upper compartment 
of Transwell chambers for a further $24 \mathrm{~h}$. To accelerate endothelial dysfunction, the culture medium was replaced with serum-free M199 containing $10 \mathrm{ng} / \mathrm{mL}$ human recombinant tumor necrosis factor-alpha (TNF- $\alpha$ ) (PeproTech, Rocky Hill, NJ, USA) and $40 \mu \mathrm{g} / \mathrm{mL}$ oxidized low-density lipoprotein (ox-LDL) (Yiyuan Biotech, Guangzhou, People's Republic of China). ${ }^{35,36}$ Finally, the expression of CD44 receptors on HUVEC monolayer and the permeability of HUVEC monolayer after stimulation with TNF- $\alpha$ and ox-LDL for $12 \mathrm{~h}$ were analyzed to validate the formation of the injured HUVECs/iM $\Phi$ co-culture system.

\section{The validation tests for the injured endothelium- macrophage co-culture system}

HUVECs on the Transwell filters were harvested, washed with PBS, and incubated with fluorescein isothiocyanate (FITC)-labeled anti-human CD44 and IgG2b isotype control (BioLegend, San Diego, CA, USA), respectively, for $30 \mathrm{~min}$ at $4^{\circ} \mathrm{C}$. HUVECs were then washed three times with cold PBS, and the mean fluorescence intensity was analyzed by flow cytometry (BD FACSCalibur; BD Biosciences, San Jose, CA, USA). As a negative control, the CD44 expression level in the normal HUVECs of HUVECs/iM $\Phi$ co-culture system without TNF- $\alpha$ and ox-LDL treatment was also assayed.

For permeability measurements, $1 \mathrm{mg} / \mathrm{mL}$ FITC-labeled albumin was added into the upper compartment of the Transwell. ${ }^{37}$ Subsequently, the fluorescence intensity in the upper compartment and bottom well was measured using a fluorimeter (Jenway Ltd, Dunmow, UK) after $1 \mathrm{~h}$. The permeability $(P)$ of the HUVEC monolayer was estimated using the following equation:

$$
P(\%)=\frac{V_{1} \times C_{1}}{V_{\mathrm{u}} \times C_{\mathrm{u}}} \times 100 \%
$$

where $C_{1}$ and $C_{\mathrm{u}}$ are defined as the concentration of FITClabeled albumin in the lower compartment and upper compartment, respectively, and $V_{1}$ and $V_{\mathrm{u}}$ are defined as the volume of medium in the lower compartment and upper compartment, respectively.

Intracellular lipid dispositions in $\mathrm{M} \Phi \Phi$ were determined by Oil Red O staining. ${ }^{38}$

Cells were washed with PBS, fixed with 4\% paraformaldehyde for $15 \mathrm{~min}$, and then incubated with Oil Red $\mathrm{O}$ solution (Sigma-Aldrich Ltd.). The nuclei were also counterstained with Mayer's hematoxylin (blue) for $5 \mathrm{~min}$. After that, cells were captured by microscope (IX71; Olympus, Tokyo, Japan).

\section{¡М $\Phi$-targeting ability of nanoparticles}

In order to perform iM $\Phi$ targeting of each nanocarrier in vitro, fluorescent carbocyanine dye DiD-loaded formulations (DiD-PLN, DiD-s-rHDL, DiD-PLGA-rHDL, DiDHA-(E)-PLGA-rHDL, and DiD-HA-(C)-PLGA-rHDL) were prepared as described, respectively, except for the replacement of ST by DiD.

To simulate the hydrodynamics of atherosclerotic plaque, the d-flow was created by placing the injured HUVECs/iM $\Phi$ co-culture Transwell chambers on a platform shaker (LabnetProBlot; Labnet International, Edison, NJ, USA) with parameters of $\pm 7^{\circ}$ rotating angle and $0.5 \mathrm{~Hz}$ frequency..$^{21,39}$ After adding $2 \mathrm{mg} / \mathrm{mL}$ HAase to the lower compartment of Transwell chambers, five different DiD-loaded nanoparticles diluted by fresh serum medium were added into the upper compartment, and incubated with cells for $24 \mathrm{~h}$ at $37^{\circ} \mathrm{C}$. The localization of various DiD-loaded nanoparticles in iM $\Phi$ was then investigated with confocal laser scanning microscope. iM $\Phi$ in the lower compartment was washed three times with PBS, and fixed with 4\% paraformaldehyde for $15 \mathrm{~min}$. Afterwards, cell nuclei were stained with 4',6-diamidino2-phenylindole (DAPI) before the fluorescence imaging by confocal laser scanning microscope (Leica TCS SP5; Leica Microsystems, Wetzlar, Germany). Meanwhile, the DiD fluorescence intensity of iM $\Phi$ in the lower compartment was quantified by flow cytometry. Cells untreated with fluorescent DiD-based agents were used as a negative control.

\section{Multistage-targeting mechanism of nanoparticles}

To test whether the targeting ability of HA-(C)-PLGA-rHDL toward $\mathrm{iM} \Phi$ was firstly mediated by CD44 receptors on the surface of injured endothelium, damaged HUVECs in the upper compartment of co-culture Transwell chambers were pretreated with $10 \mathrm{mg} / \mathrm{mL}$ free HA polymer (hydrated overnight in serum-free medium) for $1 \mathrm{~h}$ before incubation with DiD-loaded formulations. Confocal laser scanning microscopy (CLSM) and flow cytometry were then conducted to examine the DiD uptake by $\mathrm{iM} \Phi$ after $24 \mathrm{~h}$.

To study whether the uptake of HA-(C)-PLGA-rHDL by iM $\Phi$ was finally dependent on SR-BI-mediated endocytosis, $100 \mu \mathrm{M}$ BLT-1, a chemical inhibitor of SR-BI, was added into the lower compartment for $2 \mathrm{~h}$ prior to the addition of DiD-loaded nanoparticles. ${ }^{40}$ The DiD fluorescence intensity of iM $\Phi$ after $24 \mathrm{~h}$ was determined by combined CLSM and flow cytometry.

The iM $\Phi$-targeting efficacy of HA-(C)-PLGA-rHDL was also investigated in the absence of HAase in the lower compartment of Transwell chambers. After incubation with 
DiD-based agents for $24 \mathrm{~h}$, the DiD uptake by iM $\Phi$ was examined by CLSM and flow cytometric analysis, respectively.

\section{Cellular cholesterol efflux}

NBD-cholesterol as a fluorescent cholesterol analog mimicking the behavior of natural cholesterol was utilized to study and compare the cholesterol efflux capacities of five different blank nanocarriers. ${ }^{41}$ Firstly, iM $\Phi$ was cultured in complete M199 medium for $24 \mathrm{~h}$ under $5 \% \mathrm{CO}_{2}$ at $37^{\circ} \mathrm{C}$, and further starved in serum-free media for $2 \mathrm{~h}$, followed by the incubation with serum-free media containing NBD-cholesterol $(1 \mu \mathrm{g} / \mathrm{mL})$ for an additional $4 \mathrm{~h}$. After discarding the media, cells were washed three times with PBS and exposed to $50 \mu \mathrm{g} / \mathrm{mL}$ of different blank nanocarriers (PLN, s-rHDL, PLGA-rHDL, HA-(E)-PLGA-rHDL, and HA-(C)-PLGArHDL) pretreated with or without HAase. The efflux media were collected at different time points until the equilibrium of cholesterol efflux was established, and centrifuged at $4^{\circ} \mathrm{C}$ for $5 \mathrm{~min}$ to remove floating cells. Meanwhile, at the selected time intervals, iM $\Phi$ was lysed by $0.1 \%$ Triton X-100 buffer for $30 \mathrm{~min}$, and the supernatant of cell lysates was collected after centrifugation at $12,000 \mathrm{rpm}$ for $15 \mathrm{~min}$. The fluorescence intensity of NBD-cholesterol in efflux media $\left(F_{\mathrm{m}}\right)$ and cell lysates $\left(F_{\mathrm{c}}\right)$ was measured using a microplate reader, respectively. According to the formula $F_{\mathrm{m}} /\left(F_{\mathrm{m}}+F_{\mathrm{c}}\right) \times 100$, the percentage of cholesterol efflux for each cholesterol acceptor at various time points was calculated, and then the cholesterol efflux-time curves of blank nanocarriers pretreated with or without HAase were graphed.

At the steady state of cholesterol efflux, cells incubated with various blank nanocarriers pretreated with or without HAase were rinsed three times with PBS and fixed with 4\% paraformaldehyde, the cell nuclei were stained with DAPI, and then CLSM was employed to visualize the intracellular residual NBD-cholesterol.

\section{Animals and model}

New Zealand White (NZW) rabbits (male, 2.3-2.5 kg) were purchased from the Nanjing Jinling Rabbit Center (Nanjing, People's Republic of China). The animal experiments were approved by the Institutional Animal Care and Use Committee of China Pharmaceutical University. All care and handling of animals in this research were carried out in compliance with the National Institute of Health Guide for the Care and Use of Laboratory Animals. Atherosclerotic NZW rabbit model was established through combining endothelial injury and high-fat diets according to the method developed in our previous studies. ${ }^{13,17}$

\section{In vivo atherosclerotic $\mathrm{i} M \Phi$-targeting property \\ Ex vivo imaging}

For ex vivo fluorescence imaging, near-infrared fluorescent dye DiR-loaded formulations (DiR-PLN, DiR-s-rHDL, DiR-PLGA-rHDL, DiR-HA-(E)-PLGA-rHDL, and DiR-HA(C)-PLGA-rHDL) were prepared as described, respectively, except for the replacement of ST by DiR. Atherosclerotic NZW rabbits were respectively administrated with DiRloaded nanoparticles at a DiR dose of $0.3 \mathrm{mg} / \mathrm{kg}$ via the auris edge vein. Moreover, free $\operatorname{DiR}(0.3 \mathrm{mg} / \mathrm{kg})$ was also injected into atherosclerotic NZW rabbits as a control. After $4 \mathrm{~h}$, all rabbits were subjected to euthanasia, and their livers and aortic trees were carefully excised. Each liver and aortic tree was then captured by imaging system ORCA-R2 (Hamamatsu Photonics, Hamamatsu, Japan), and the obtained images were analyzed using the HCImage LiveSoftware.

\section{Fluorescence localization of nanoparticles in atherosclerotic aortic roots}

In addition to the atherosclerotic NZW rabbits, normal NZW rabbits were treated with DiR-HA-(C)-PLGA-rHDL as another control group. At $4 \mathrm{~h}$ after injection, all freshly frozen aortic roots were segmented into $5 \mu \mathrm{m}$-thick sections. The sections were initially incubated with 5\% normal goat serum in $0.01 \%$ Triton $\mathrm{X}-100$ buffer at room temperature for $1 \mathrm{~h}$, subsequently stained with mouse anti-rabbit anti-CD68 antibodies (Abcam, Cambridge, UK) as primary antibody at a 1/100 dilution for $12 \mathrm{~h}$, and incubated with FITC-conjugated anti-mouse antibodies (Jackson Immuno Research, West Grove, PA, USA) as secondary antibody to visualize iM $\Phi$ in the aortic roots. After being washed three times with PBS buffer $(0.01 \%$ Triton X-100), cell nuclei of all sections were stained with DAPI, and observed under a confocal laser scanning microscope immediately. Dye accumulation and retention in organs was evaluated by calculating contrast index values using instrument software.

\section{In vivo anti-atherogenic efficacies in model animals}

In accordance with the treatment protocol published in our previous research, 42 NZW rabbits were randomly divided into the following seven groups $(n=6)$ : (i) positive control group without any treatment (model group); (ii) ST-Capsule; (iii) ST-PLN; (iv) ST-s-rHDL; (v) ST-PLGA-rHDL; (vi) STHA-(E)-PLGA-rHDL; and (vii) ST-HA-(C)-PLGA-rHDL. After being fed with high-fat diet for 8 weeks, the NZW rabbits in the ST-Capsule group were orally administrated 
a dose of $8 \mathrm{mg} / \mathrm{kg}$, while the other NZW rabbits in the six ST nanoparticles groups were intravenously administrated via the marginal ear vein a dose of $0.4 \mathrm{mg} / \mathrm{kg}$, every other day, for an additional 8 weeks. Normal NZW rabbits $(n=6)$ were taken as the negative control group (normal group). Then, all groups were subjected to the following assessments so as to evaluate anti-atherogenic efficacies of different preparations.

\section{Oil Red $O$ staining for plaque area analysis}

Plaque area is commonly evaluated by Oil Red $\mathrm{O}$ staining of lipid deposits. ${ }^{42}$ The entire aortas of rabbits in all groups were dissected free, and the adventitial fat was removed carefully. After being opened longitudinally with the luminal surface toward the outside, aortas were stained with Oil Red $\mathrm{O}$ solution for $4 \mathrm{~h}$ at $25^{\circ} \mathrm{C}$, and then differentiated by $60 \%$ propylene glycol until the atherosclerotic plaque was stained red and the arterial wall was white. All stained aortas were captured by EOS 5D Mark III (Canon, Tokyo, Japan). Finally, the lesions positive staining (\%), defined as percent lesion area per total aorta zone, was quantified by Image-Pro Plus 6.0 to evaluate the extent of atherosclerosis after treatment.

\section{Immunohistochemistry for iM $\Phi$ expression}

All paraformaldehyde-fixed and paraffin-embedded aortas were sectioned into $4 \mathrm{~mm}$-thick specimens, dewaxed, and rehydrated. ${ }^{43}$ Firstly, endogenous peroxidase activity was quenched by $3 \%$ hydrogen peroxide in methanol for $30 \mathrm{~min}$, and nonspecific antibody reactivity was blocked by incubating with $5 \%$ normal goat serum in $0.01 \%$ Triton X-100 buffer for $1 \mathrm{~h}$. Subsequently, iM $\Phi$ in specimens was identified with mouse anti-rabbit anti-CD68 antibodies as primary antibody at a $1 / 100$ dilution for $12 \mathrm{~h}$. Then, specimens were incubated with secondary antibody for $1 \mathrm{~h}$, stained with 3,3'diaminobenzidine tetrahydrochloride (Sigma-Aldrich Ltd.), and counterstained with Weigert's hematoxylin. Ultimately, the Image-Pro Plus 6.0 software was used to assess the positive staining $(\%)$ of $\mathrm{iM} \Phi$.

\section{Gelatinolytic activity assay}

To determine the MMP activity in the atherosclerotic lesion, cryosections of aortic arch from each group were examined by an in situ zymography fluorescence staining kit (Genmed Scientifics Inc., Shanghai, People's Republic of China). The pre-melted gelatin was firstly mixed with preheated staining solution containing DAPI to counterstain the cell nuclei. Then, the mixture was added into cryosections, followed by coverslips. After gelling of the gelatin at $4^{\circ} \mathrm{C}$, slides were incubated in a dark chamber at $37^{\circ} \mathrm{C}$ for $1 \mathrm{~h}$. Ultimately, fluorescence gelatinolytic activities of all slides were detected using an Image Xpress Ultra automated-confocal microscope (Molecular Devices, Sunnyvale, USA), and the intensity of green fluorescence (without selecting autofluorescent elastic fibers) was measured by using the Image-Pro Plus 6.0 software.

\section{Statistical analysis}

Each experiment was performed at least in triplicate with independent samples. Statistical analysis was performed using unpaired Student's $t$-test for comparisons of two groups, while one-way analysis of variance was used for multiple-group comparisons. Data are expressed as mean \pm standard deviation unless otherwise indicated. A $P$-value of less than 0.05 was considered statistically significant.

\section{Results}

\section{In vitro characterizations}

Mean size, zeta potential, EE, DL, and HA conjugation efficiency

The physicochemical characteristics of five different nanoparticle groups are given in Table 1. After incubation with apoA-I, the mean size of ST-PLN increased to $120.9 \pm 1.9 \mathrm{~nm}$, and zeta potential decreased from $27.83 \pm 0.44$ to $19.36 \pm 0.43 \mathrm{mV}$. Compared with ST-PLGA-rHDL, ST-HA-(E)-PLGA-rHDL

Table I Particle size, zeta potential, EE, DL, and $\mathrm{BE}_{\mathrm{HA}}$ results of five different ST-loaded nanoparticles

\begin{tabular}{|c|c|c|c|c|c|}
\hline & ST-PLN & ST-s-rHDL & ST-PLGA-rHDL & $\begin{array}{l}\text { ST-HA-(E)- } \\
\text { PLGA-rHDL }\end{array}$ & $\begin{array}{l}\text { ST-HA-(C)- } \\
\text { PLGA-rHDL }\end{array}$ \\
\hline Particle size $(\mathrm{nm})$ & $109.4 \pm 2.6$ & $124.5 \pm 1.9$ & $120.9 \pm 1.7$ & $131.8 \pm 2.7$ & $138.2 \pm 2.3$ \\
\hline Zeta potential $(\mathrm{mV})$ & $27.83 \pm 0.44$ & $-30.67 \pm 0.71$ & $19.36 \pm 0.43$ & $-22.13 \pm 0.41$ & $-28.38 \pm 0.52$ \\
\hline EE (\%) & $91.92 \pm 0.54$ & $90.60 \pm 0.58$ & $91.11 \pm 0.45$ & $90.73 \pm 0.52$ & $90.64 \pm 0.43$ \\
\hline $\mathrm{DL}(\%)$ & $6.05 \pm 0.19$ & $5.02 \pm 0.37$ & $5.79 \pm 0.21$ & $5.18 \pm 0.34$ & $5.03 \pm 0.32$ \\
\hline $\mathrm{BE}_{\mathrm{HA}}(\%)$ & - & - & - & $25.33 \pm 0.32$ & $34.51 \pm 0.37$ \\
\hline
\end{tabular}

Notes: Data are expressed as the mean \pm standard deviation, $n=3$. “-” indicates data not measured.

Abbreviations: EE, entrapment efficiency; DL, drug loading; $\mathrm{BE}_{\mathrm{HA}}$, HA-binding efficiency; ST, simvastatin; PLN, PLGA-lipid nanoparticle; s-rHDL, spherical rHDL; PLGA, poly (lactic-co-glycolic acid); rHDL, reconstituted high-density lipoprotein; HA, hyaluronan; (E), electrostatically attached; (C), covalently attached. 
constructed by the electrostatic absorption of HA exhibited a slightly ascended size of $131.8 \pm 2.7 \mathrm{~nm}$, and the zeta potential shifted from positive values to negative values $(-23.13 \pm 0.41 \mathrm{mV})$. In contrast to ST-HA-(C)-PLGA-rHDL synthesized by HA covalent binding showed a larger diameter $(138.2 \pm 2.3 \mathrm{~nm})$ and an increased absolute value of negative zeta potential $(-28.38 \pm 0.52 \mathrm{mV})$. This observation was also consistent with the distinct $\mathrm{BE}_{\mathrm{HA}}$ value of ST-HA-(E)-PLGArHDL $(25.33 \% \pm 0.32 \%)$ and ST-HA-(C)-PLGA-rHDL $(34.51 \% \pm 0.37 \%)$. EE was more than $90 \%$ for these four preparations, and DL was $6.05 \% \pm 0.19 \%, 5.79 \% \pm 0.21 \%$, $5.18 \% \pm 0.34 \%$, and $5.03 \% \pm 0.32 \%$, respectively. Moreover, the mean sizes and zeta potentials of ST-s-rHDL were similar to the results reported in our previous study. ${ }^{10}$

\section{Morphology}

Microstructures of different ST-loaded nanocarriers were visualized by TEM (Figure 2A). ST-s-rHDL is presented as black spherical spots in accordance with our previous study. While ST-PLN and ST-PLGA-rHDL appeared as the obvious core-shell structures with a white spot surrounded by a gray rim, the white spherical core had a similar morphology as ST-PN, which indicated that the spherical PLGA core was coated by a lipid bilayer or apoA-I. TEM revealed the similar
A

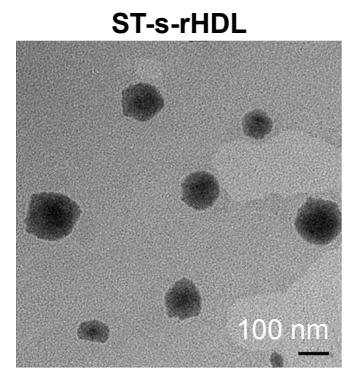

ST-PLGA-rHDL

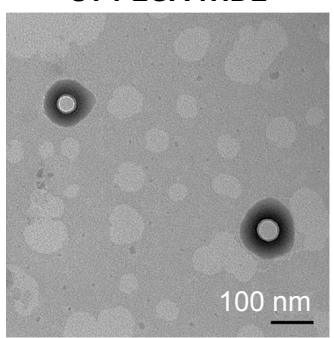

B

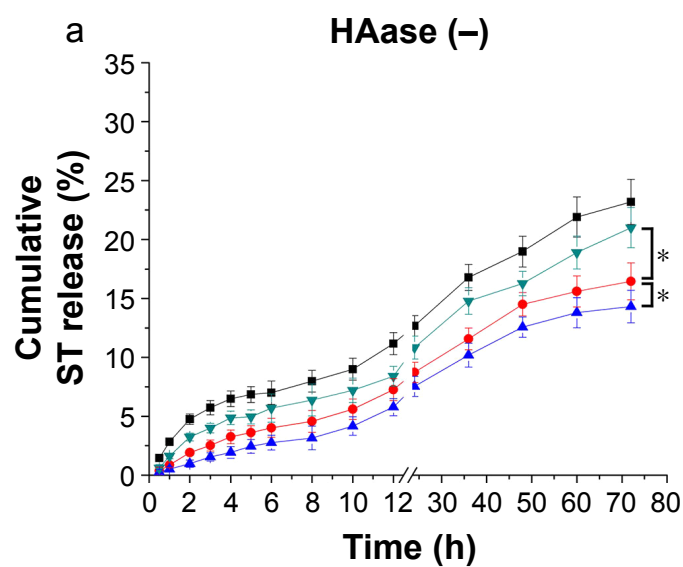

ST-PN

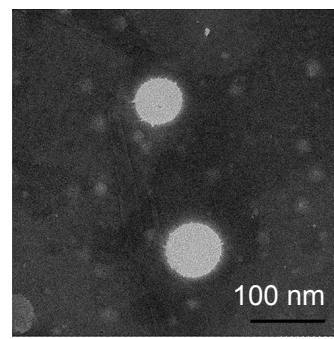

ST-HA-(E)-PLGA-rHDL

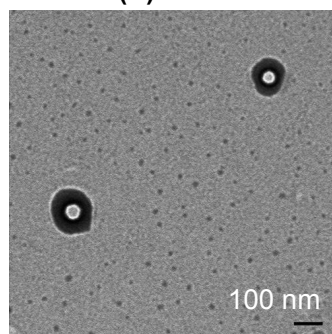

0
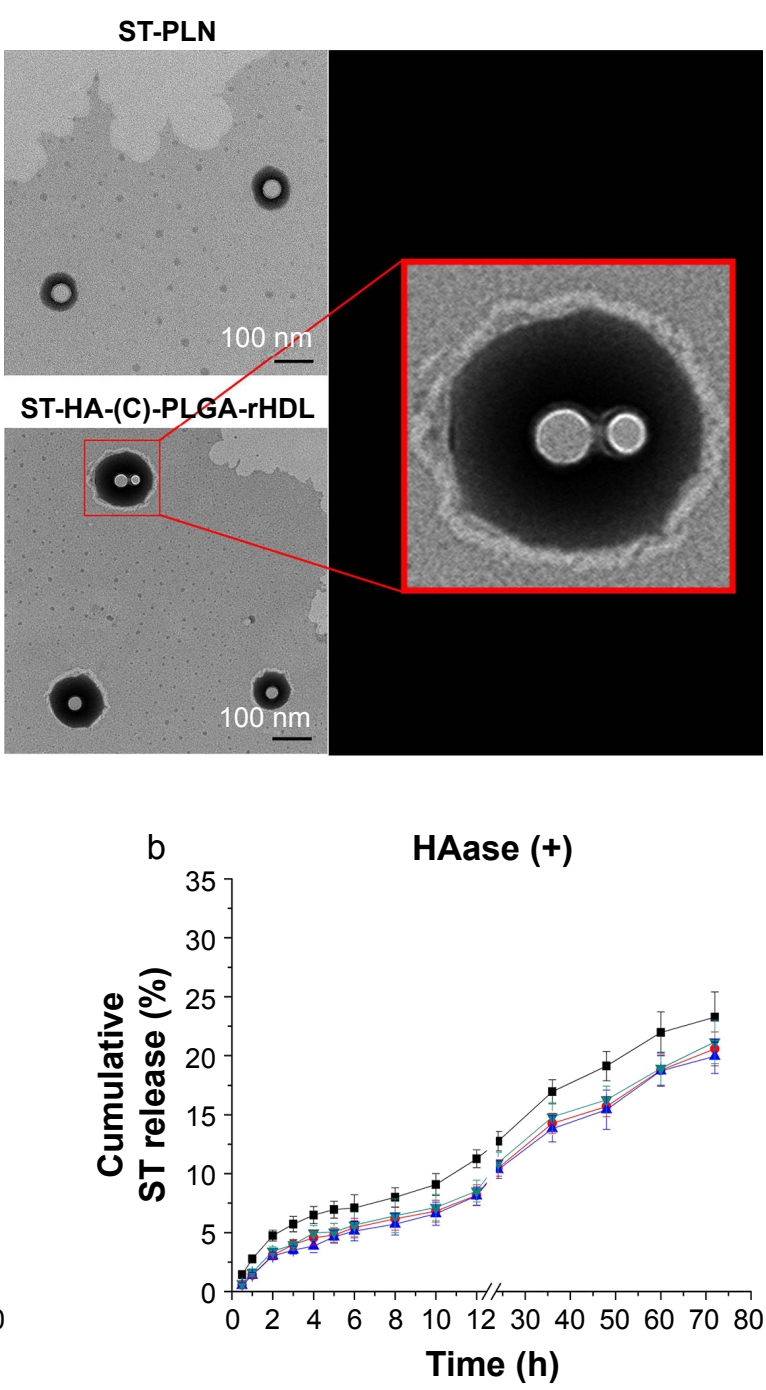

$-\mathrm{ST}-\mathrm{PLN} \longrightarrow \mathrm{ST}-\mathrm{PLGA}-\mathrm{rHDL} \longrightarrow \mathrm{ST}-\mathrm{HA}-(\mathrm{E})-\mathrm{PLGA}-\mathrm{rHDL} \leadsto \mathrm{ST}-\mathrm{HA}-(\mathrm{C})-\mathrm{PLGA}-\mathrm{rHDL}$

Figure 2 TEM image and in vitro drug release profiles both in the absence and presence of HAase.

Notes: TEM (A) of ST-PN, ST-PLN, ST-s-rHDL, ST-PLGA-rHDL, ST-HA-(E)-PLGA-rHDL, and ST-HA-(C)-PLGA-rHDL. In vitro drug release profiles (B) both in the absence (a) and presence (b) of HAase. $* P<0.05$. Data are expressed as the mean $\pm S D(n=4)$.

Abbreviations: TEM, transmission electron microscopy; HAase, hyaluronidase; ST, simvastatin; PN, PLGA nanoparticle; PLN, PLGA-lipid nanoparticle; s-rHDL, spherical rHDL; PLGA, poly (lactic-co-glycolic acid); rHDL, reconstituted high-density lipoprotein; HA, hyaluronan; (E), electrostatically attached; (C), covalently attached; $\mathrm{SD}$, standard deviation. 
core-multishell structures of ST-HA-(E)-PLGA-rHDL and ST-HA-(C)-PLGA-rHDL, exhibiting a core-shell structural ST-PLGA-rHDL surrounded by a white brim through HA decoration. In particular, the white HA layer of ST-HA-(C)PLGA-rHDL was more distinguishable with a better roundness compared with ST-HA-(E)-PLGA-rHDL.

\section{In vitro release in the HAase environment}

Patterns of release of ST from different ST-loaded nanocarriers evaluated with or without HAase are shown in Figure 2B. The cumulative release percent of ST-PLN and ST-PLGArHDL within $72 \mathrm{~h}$ in the absence of HAase was about $23.19 \% \pm 1.91 \%$ and $21.00 \% \pm 1.71 \%$, respectively. Their release behaviors were almost unchanged in the presence of HAase. In the absence of HAase, the release of ST from
ST-HA-(E)-PLGA-rHDL was about $16.46 \% \pm 1.57 \%$ after $72 \mathrm{~h}$, while that from ST-HA-(C)-PLGA-rHDL was about $14.32 \% \pm 1.39 \%$. By comparison, the rates of release of ST from both ST-HA-(E)-PLGA-rHDL and ST-HA-(C)-PLGArHDL were much higher in the case of HAase-catalyzed degradation $(20.58 \% \pm 1.43 \%$ and $19.95 \% \pm 1.45 \%)$, which were almost identical to that of ST-PLGA-rHDL with or without HAase.

\section{In vitro iM $\Phi$-targeting property and multistage-targeting mechanism Injured endothelium-macrophage co-culture system establishment}

As shown in Figure 3A, an injured endothelium-macrophage co-culture system mimicking the in vivo impaired vascular
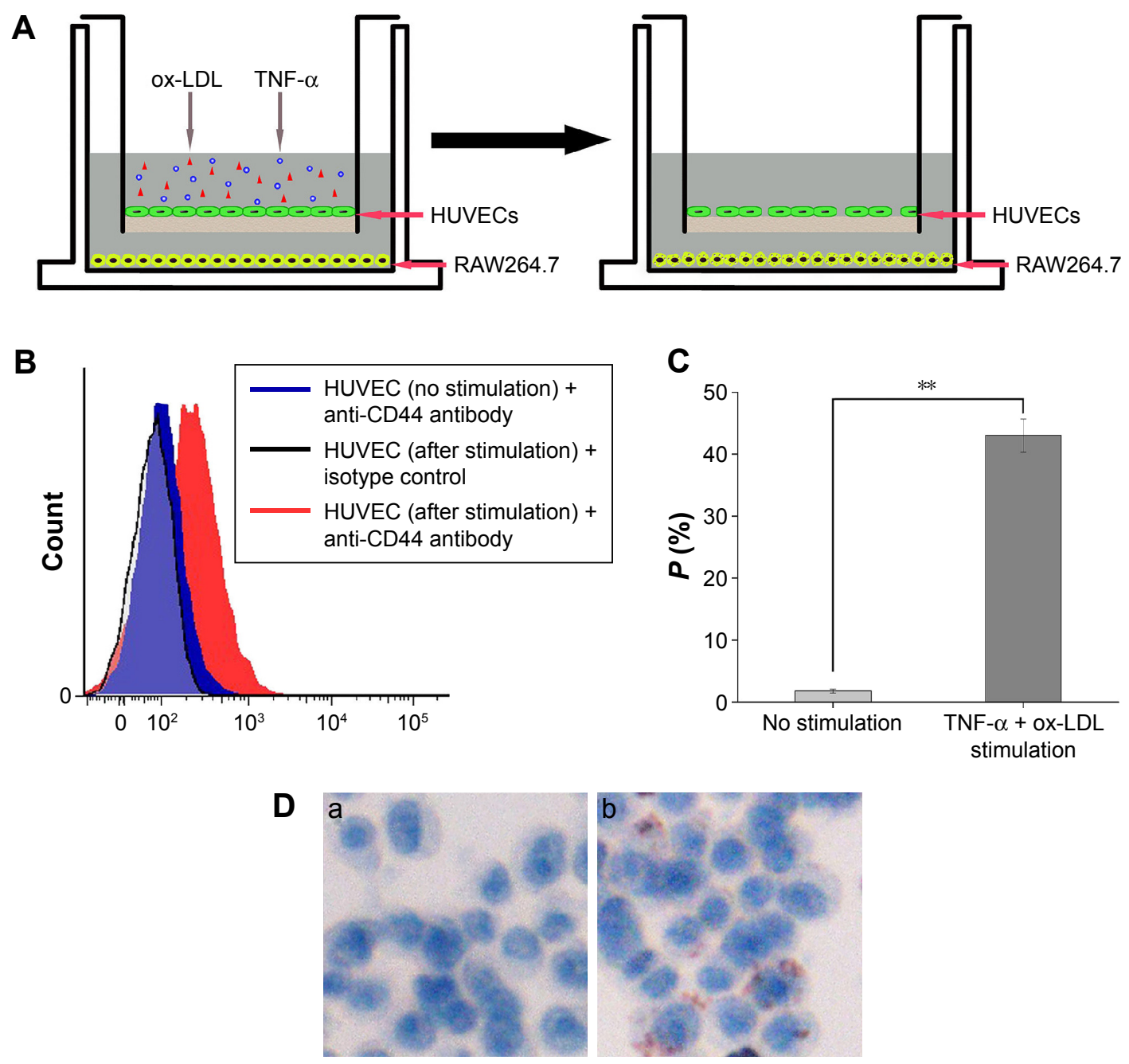

Figure 3 Establishment of injured endothelium-macrophage co-culture system.

Notes: Schematic illustration (A) of fabrication procedure of injured endothelium/macrophage co-culture system in Transwell chambers. Flow cytometry analysis (B) for CD44 expression in HUVECs of HUVECs/iM $\Phi$ co-culture system with and without costimulation from TNF- $\alpha$ and ox-LDL. Permeability (C) of HUVEC monolayer in HUVECs/iMФ co-culture system with and without costimulation from TNF- $\alpha$ and ox-LDL. Oil Red O lipid staining (D) of HUVECs/iM $\Phi$ co-culture system without (a) and with (b) costimulation from TNF- $\alpha$ and ox-LDL (Images captured at magnification $\times 400$ ). $* * P<0.01$. Data are expressed as the mean \pm SD ( $n=3$ ).

Abbreviations: HUVECs, human umbilical vein endothelial cells; iM $\Phi$, RAW264.7 macrophage cell line; $P$, permeability; TNF- $\alpha$, human recombinant tumor necrosis factoralpha; ox-LDL, oxidized low-density lipoprotein; SD, standard deviation. 
endothelial barrier of atherosclerotic plaque was first developed in Transwell chambers upon exposure of the cells to TNF- $\alpha$ and ox-LDL. ${ }^{35,36}$

The CD44 receptor levels of HUVECs after stimulation with TNF- $\alpha$ and ox-LDL for $12 \mathrm{~h}$ were evaluated by flow cytometry. After being stained with FITC-labeled anti-human CD44, all tested cells exhibited significantly higher binding activities to FITC-labeled anti-human CD44 than that of the isotype control (Figure 3B), indicating that there was an expression of CD44 receptors in the HUVECs treated with TNF- $\alpha$ and ox-LDL. Furthermore, compared with the normal HUVECs, the CD44 expression level of HUVECs after stimulation with TNF- $\alpha$ and ox-LDL was markedly augmented, which was also in agreement with a previous report. ${ }^{44}$

Regarding permeability experiment (Figure 3C), there was a statistically significant increase in the permeability $(P \%)$ of the HUVEC monolayer after stimulation in contrast to normal HUVEC monolayer (no treatment) $(P<0.01)$.

Moreover, compared with normal control group, an increased lipid deposition in the $\mathrm{iM} \Phi$ of endotheliummacrophage co-culture system after treatment with TNF- $\alpha$ and ox-LDL was determined (Figure 3D), which was probably due to the uptake of ox-LDL transmigrating through the injured HUVEC monolayer with enhanced permeability, and in accord with the important pathologic process of atherosclerosis. ${ }^{45}$ All these results revealed the successful establishment of an injured endothelium-macrophage co-culture model.

\section{iМФ-targeting property and multistage-targeting mechanism}

Following $24 \mathrm{~h}$ of exposure to the blank nanocarriers with the concentrations ranging from 20 to $500 \mu \mathrm{g} / \mathrm{mL}$, no obvious cytotoxicity against HUVECs and $\mathrm{iM} \Phi$ was observed (Figure S1). The results meant that all the blank nanocarriers $(20-500 \mu \mathrm{g} / \mathrm{mL})$ were safe and nontoxic for the cell studies.

The iM $\Phi$-targeting abilities of different nanocarriers were investigated in the injured HUVECs/iM $\Phi$ co-culture system under d-flow condition. DiD was utilized as a fluorescent probe for CLSM and flow cytometry. After incubation with DiD-based agents for $24 \mathrm{~h}$, visual examination by CLSM indicated that the strongest fluorescence was observed in the iM $\Phi$ of DiD-HA-(C)-PLGA-rHDL group as expected, and the red fluorescence intensities of the other four nanoparticles were increased gradually in the following order: DiD-PLN, DiD-s-rHDL, DiD-PLGA-rHDL, and DiDHA-(E)-PLGA-rHDL, respectively (Figure 4A). Quantitative evaluation by flow cytometry analysis further verified the significantly distinct uptake by $\mathrm{iM} \Phi$ among different nanoparticles under d-flow (Figure 5Aa and Ba). Cellular uptake of DiD-s-rHDL amounted to 1.52-fold higher than that of DiD-PLN $(P<0.01)$; compared with DiD-s-rHDL, a slightly increased cellular uptake occurred in DiD-PLGArHDL with no significant difference; regarding response to DiD-HA-(E)-PLGA-rHDL, cellular uptake was significantly augmented compared with DiD-PLGA-rHDL (1.79-fold, $P<0.01)$; iM $\Phi$ incubated with DiD-HA-(C)-PLGA-rHDL displayed 1.35-fold higher fluorescence than that of DiDHA-(E)-PLGA-rHDL $(P<0.01)$.

To validate the specificity of HA-(C)-PLGA-rHDL accumulation in injured endothelium through the CD44 receptor during the initial stage of multistage-targeting drug delivery, damaged HUVECs in the co-culture Transwell chambers were pretreated with a saturable amount of free HA to block the CD44 receptors. As shown in Figure 4B, the lower fluorescence was observed in both the $\mathrm{iM} \Phi$ incubated with DiD-HA-(E)-PLGA-rHDL and that with DiD-HA-(C)PLGA-rHDL after treatment with free HA, compared with the untreated ones (Figure 4A), while the free HA pretreatment had no effect on the $\mathrm{iM} \Phi$ uptake of DiD-PLN, DiD-srHDL, and DiD-PLGA-rHDL. These results were verified by quantitative flow cytometric analysis (Figure $5 \mathrm{Ab}$ and $\mathrm{Bb}$ ). In particular, in comparison with the cells without HA treatment (Figure 5Aa and $\mathrm{Ba}$ ), mean fluorescence of DiD-HA-(E)PLGA-rHDL was reduced by nearly $60 \%$ in cells treated with excess HA. This phenomenon was more obviously seen in the DiD-HA-(C)-PLGA-rHDL group. The cellular uptake of DiD-HA-(C)-PLGA-rHDL after excess HA treatment underwent a notable reduction (approximately 78\%) compared to the untreated group $(P<0.01)$.

To verify the ultimate cellular internalization of HA(C)-PLGA-rHDL after HAase degradation via SR-BImediated endocytosis, BLT-1 was applied to specifically inhibit the functions of SR-BI receptors on $\mathrm{iM} \Phi$. As shown in Figures $4 \mathrm{C}$ and $5 \mathrm{Ac}$ and $\mathrm{Bc}$, the BLT-1 significantly reduced the cellular uptake of HA-(C)-PLGA-rHDL, DiDHA-(E)-PLGA-rHDL, DiD-PLGA-rHDL, and DiD-s-rHDL by more than $50 \%$ in contrast to no inhibitor treatment (Figures 4A and 5Aa and $\mathrm{Ba}$ ), however the cellular uptake of DiD-PLN in the presence of BLT-1 was comparable to that observed without BLT-1.

To further demonstrate the effect of HAase on the iM $\Phi$ targeting efficacy of HA-(C)-PLGA-rHDL, the cellular uptake in the absence of HAase was investigated and displayed in Figures 4D and 5Ad and Bd. There was almost no difference in cellular uptake of DiD-PLN between groups treated with HAase (Figures 4A and 5Aa and $\mathrm{Ba}$ ) and without HAase, and also in the DiD-s-rHDL- and DiD-PLGA-rHDL-treated 
A

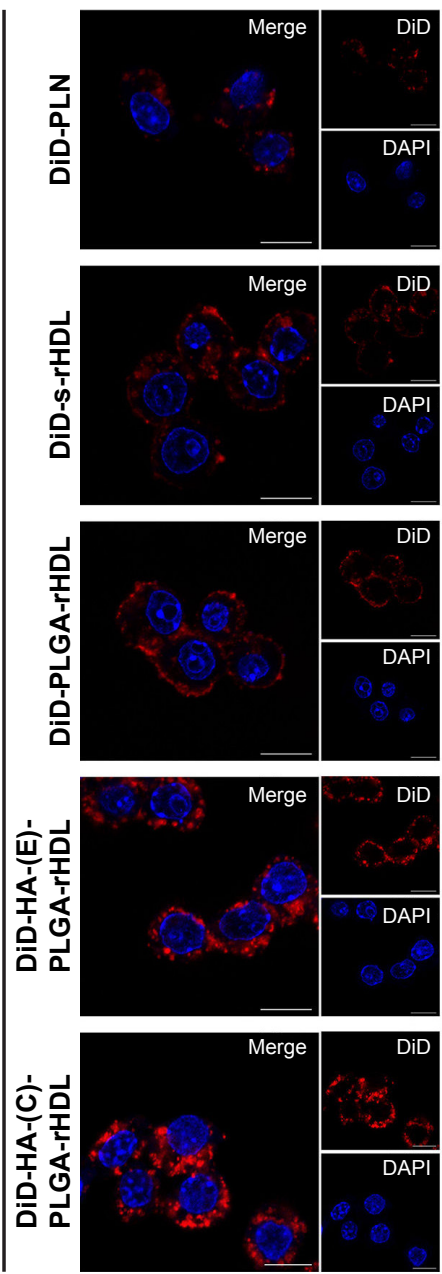

B
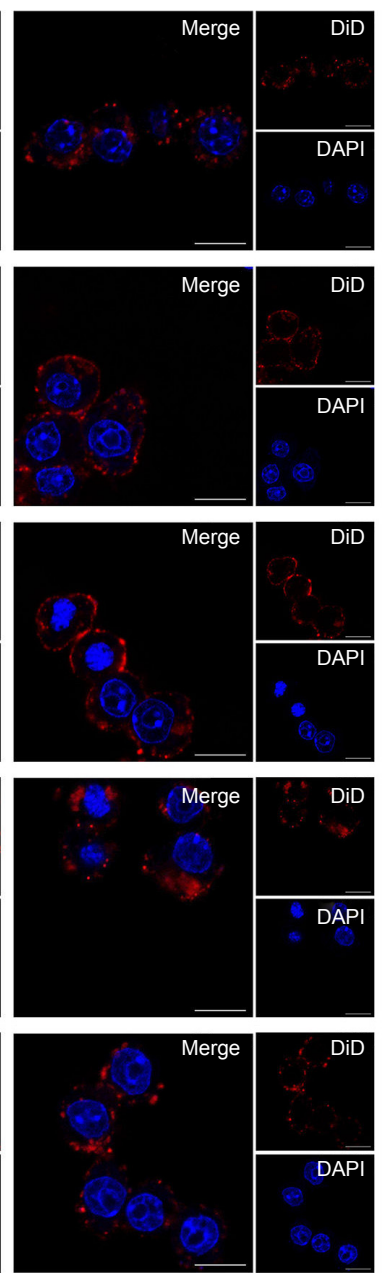

C
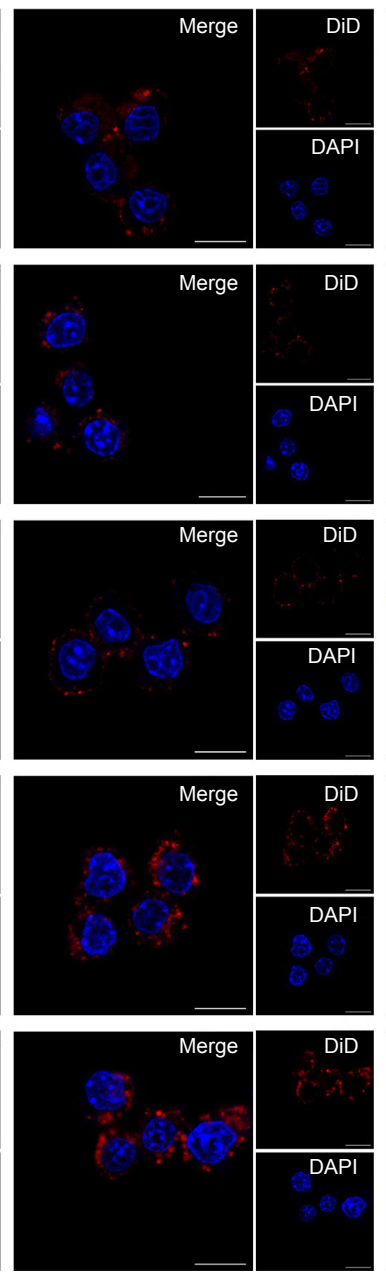

D
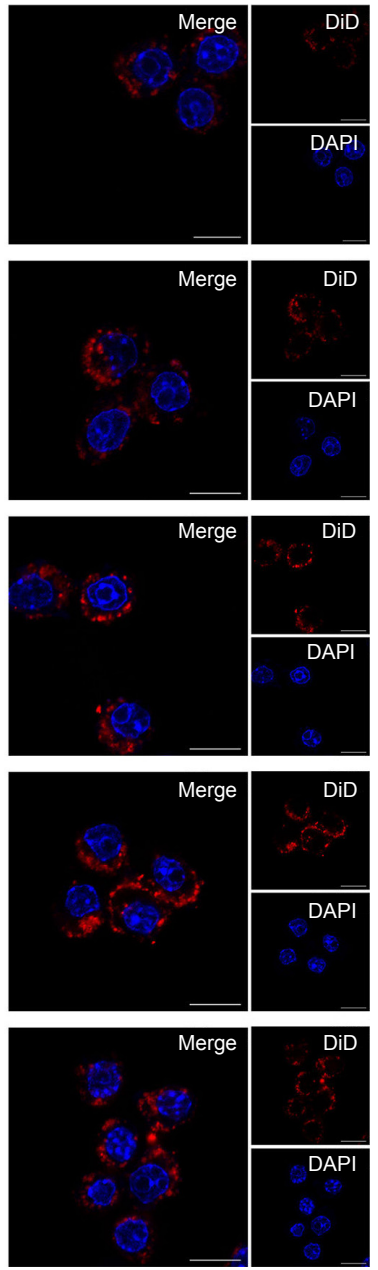

Figure 4 Cellular uptake by iMФ and multistage-targeting mechanisms investigated by CLSM.

Notes: Cellular internalization (A) of various DiD-loaded nanoparticles by iM $\Phi$ in injured HUVECs/iM $\Phi$ co-culture dynamic model after 24 h. iM $\Phi$-targeting mechanisms of various DiD-loaded nanoparticles studied by using $10 \mathrm{mg} / \mathrm{mL}$ of free HA polymer (B), SR-BI receptors inhibitor (I00 $\mu M$ of BLT-I) (C), and no HAase treatment (D). Images show the red fluorescence of DiD, the blue fluorescence of DAPI, and the merged fluorescence. Scale bars, $10 \mu \mathrm{m}$.

Abbreviations: iMФ, RAW264.7 macrophage cell line; CLSM, confocal laser scanning microscopy; DiD, I, I'-dioctadecyl-3,3,3',3'-tetramethylindodicarbocyanine perchlorate; HUVECs, human umbilical vein endothelial cells; HA, hyaluronan; SR-BI, scavenger receptor-BI; BLT-I, block lipid transport-I; HAase, hyaluronidase; DAPI, 4',6-diamidino-2phenylindole; PLN, PLGA-lipid nanoparticle; s-rHDL, spherical rHDL; PLGA, poly (lactic-co-glycolic acid); rHDL, reconstituted high-density lipoprotein; (E), electrostatically attached; (C), covalently attached.

groups, while 34\% reduction of cellular uptake was shown in DiD-HA-(E)-PLGA-rHDL-treated group compared to that treated with HAase. More obviously, the mean fluorescence of DiD-HA-(C)-PLGA-rHDL was reduced by $58 \%$ in cells without HAase in comparison with the cells in the presence of HAase (Figure 5Aa and Ba).

\section{Cellular cholesterol efflux}

Figure S2 illustrates the cholesterol efflux-time profiles of five blank nanoparticles pretreated with or without HAase, respectively. For blank nanoparticles without HAase treatment, cholesterol efflux from iM $\Phi$ increased gradually with incubation time, and so did that of nanoparticles with HAase treatment. After incubation with each of the nanocarriers for $6 \mathrm{~h}$, the percentage of cholesterol efflux from iM $\Phi$ was almost unchanged, indicating that the equilibrium state was reached.

Cells containing NBD-cholesterol, but without nanocarriers incubation, were used as a positive control, and the strongest NBD-cholesterol fluorescence was observed by CLSM. The residual fluorescent cholesterol of iM $\Phi$ after incubation with blank nanocarriers for $6 \mathrm{~h}$ was also visualized (Figure 6A). More residual fluorescent cholesterol in iMФ incubated with blank nanocarriers suggested the lesser efflux of cell cholesterol mediated by blank nanocarriers.

As shown in Figure 6B, the percentage of cholesterol efflux mediated by blank nanocarriers at the equilibrium state was quantified. Among nanocarriers with no HAase treatment, 
A
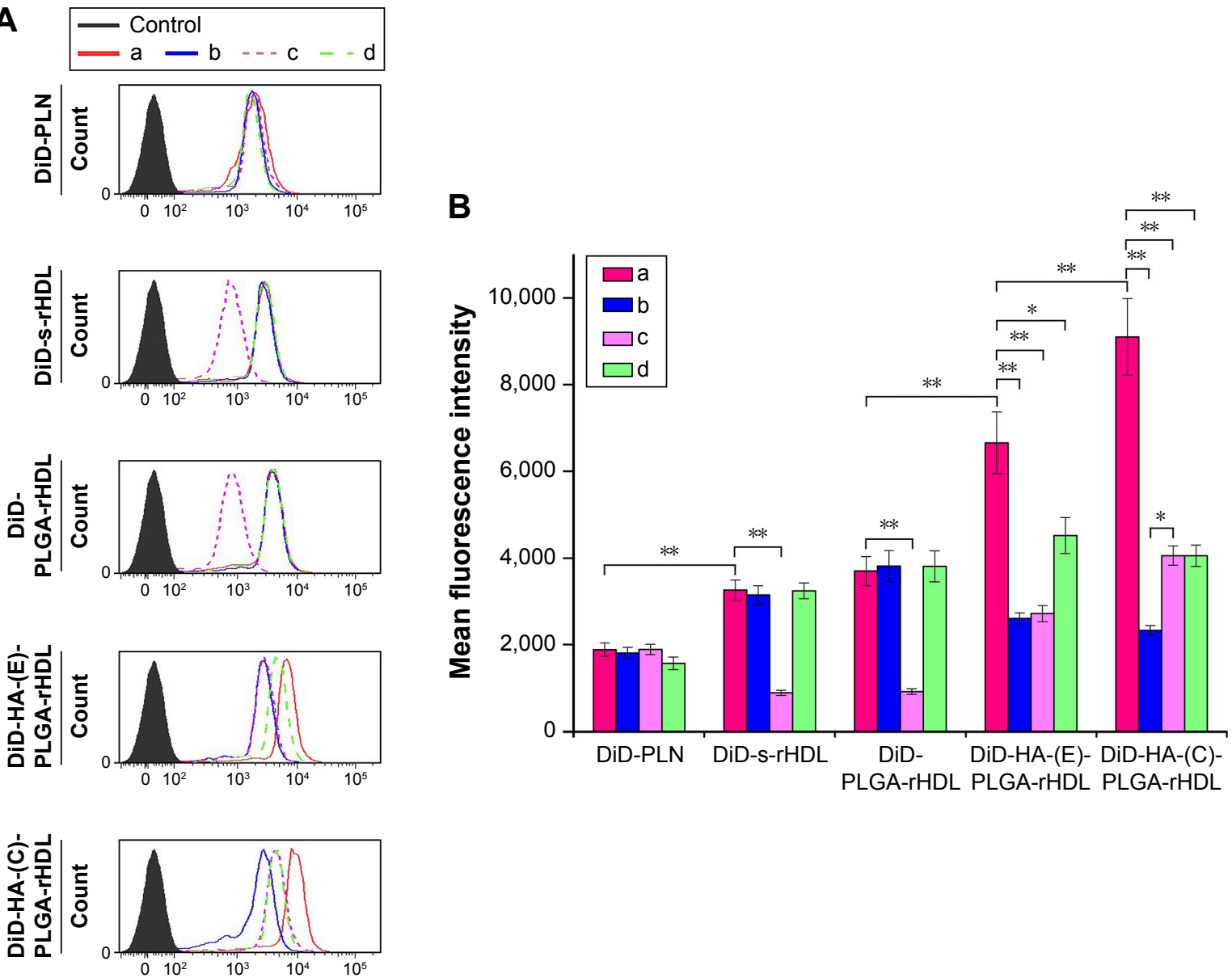

Figure 5 Cellular uptake by iM $\Phi$ and multistage-targeting mechanisms investigated by flow cytometry analysis.

Notes: Flow cytometry analysis (A) and corresponding mean fluorescence intensity (B) of various DiD-loaded nanoparticles internalized by iM $\Phi$ (a) in injured HUVECs/iM $\Phi$ co-culture dynamic model after $24 \mathrm{~h}$. iM $\Phi$-targeting mechanisms of various DiD-loaded nanoparticles studied by using $10 \mathrm{mg} / \mathrm{mL}$ of free $\mathrm{HA}$ polymer (b), SR-BI receptors inhibitor (I00 $\mu$ M of BLT-I) (c), and no HAase treatment (d). $* P<0.05$ and $* * P<0.01$. Data are expressed as the mean $\pm S D(n=4)$.

Abbreviations: iMФ, RAW264.7 macrophage cell line; DiD, I, I'-dioctadecyl-3,3,3',3'-tetramethylindodicarbocyanine perchlorate; HUVECs, human umbilical vein endothelial cells; HA, hyaluronan; SR-BI, scavenger receptor-BI; BLT-I, block lipid transport-I; HAase, hyaluronidase; SD, standard deviation; PLN, PLGA-lipid nanoparticle; s-rHDL, spherical rHDL; PLGA, poly (lactic-co-glycolic acid); rHDL, reconstituted high-density lipoprotein; (E), electrostatically attached; (C), covalently attached.

cells incubated with PLN exhibited very little cholesterol efflux, and cholesterol efflux from iM $\Phi$ mediated by s-rHDL was significantly higher than that of PLN $(P<0.05)$; the maximum cholesterol efflux (nearly $30 \%$ ) occurred at cells treated with PLGA-rHDL; compared with PLGA-rHDL, the cholesterol efflux percentage of HA-(E)-PLGA-rHDL was significantly decreased $(P<0.01)$, but slightly higher than s-rHDL group, while HA-(C)-PLGA-rHDL caused the least amount of cholesterol efflux, which was remarkably lower than that of HA-(E)-PLGA-rHDL $(P<0.01)$.

PLGA-rHDL pretreated with and without HAase generated almost identical cholesterol efflux, and the same effect was also observed in the case of PLN and s-rHDL. In contrast to treatment without HAase, both HA-(E)-PLGArHDL and HA-(C)-PLGA-rHDL with HAase pretreatment caused significantly more cholesterol efflux (nearly $30 \%$ ), which was close to the cholesterol efflux levels of
PLGA-rHDL. Attractively, the cholesterol efflux percentage of HA-(C)-PLGA-rHDL in the presence of HAase was 2.43 -fold higher than that of s-rHDL.

\section{In vivo atherosclerotic iMФ-targeting property \\ Ex vivo imaging}

The atherosclerotic-lesions-targeting efficiency of various DiR-loaded preparations was firstly evaluated in the level of arterial vessels by ex vivo imaging. As displayed in Figure 7Aa and $\mathrm{Ba}$, there was almost no fluorescence intensity at the aortic trees of free DiR groups, and the fluorescence intensity in aortic trees from DiR-PLN groups was weakest among that of DiR-loaded nanoparticles. By comparison, DiR-s-rHDL showed stronger fluorescence signals than DiR-PLN in aortic trees $(P<0.01)$, and slightly weaker signals than DiR-PLGA-rHDL with no statistically significant 
A a

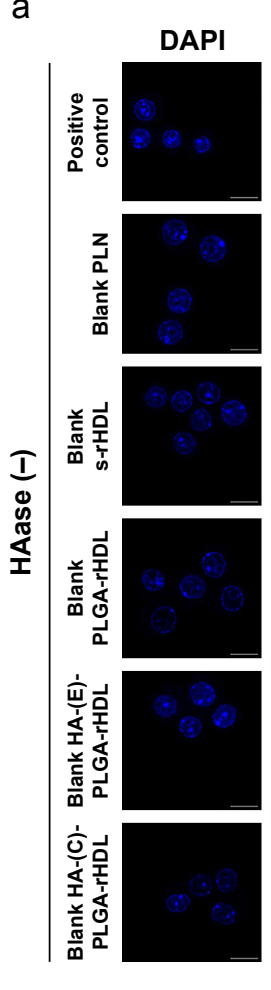

B
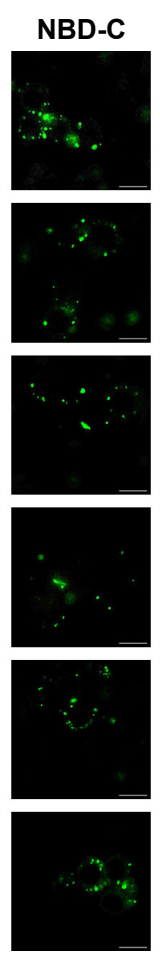

Bright-
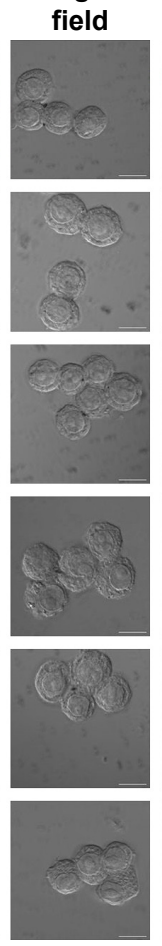

HAase (-) b
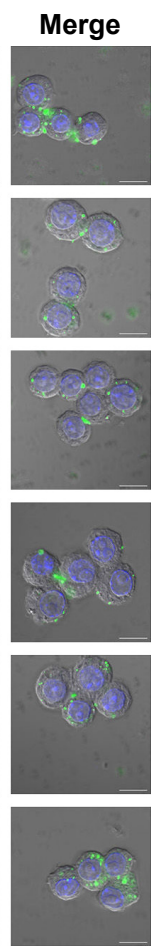

b
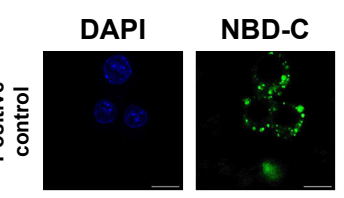

Bright-
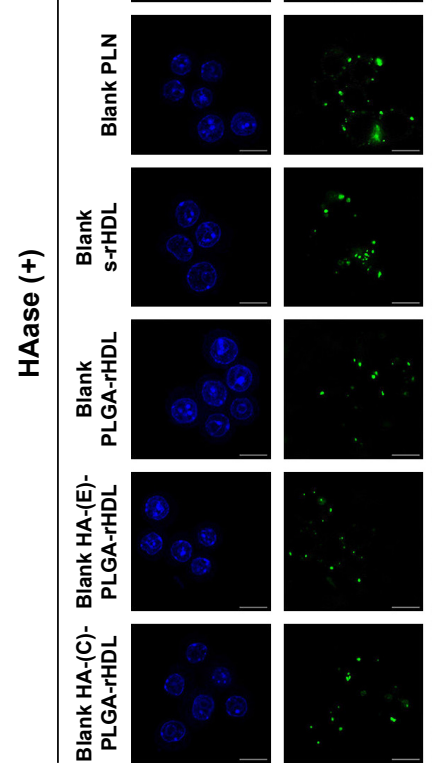

HAase (+)

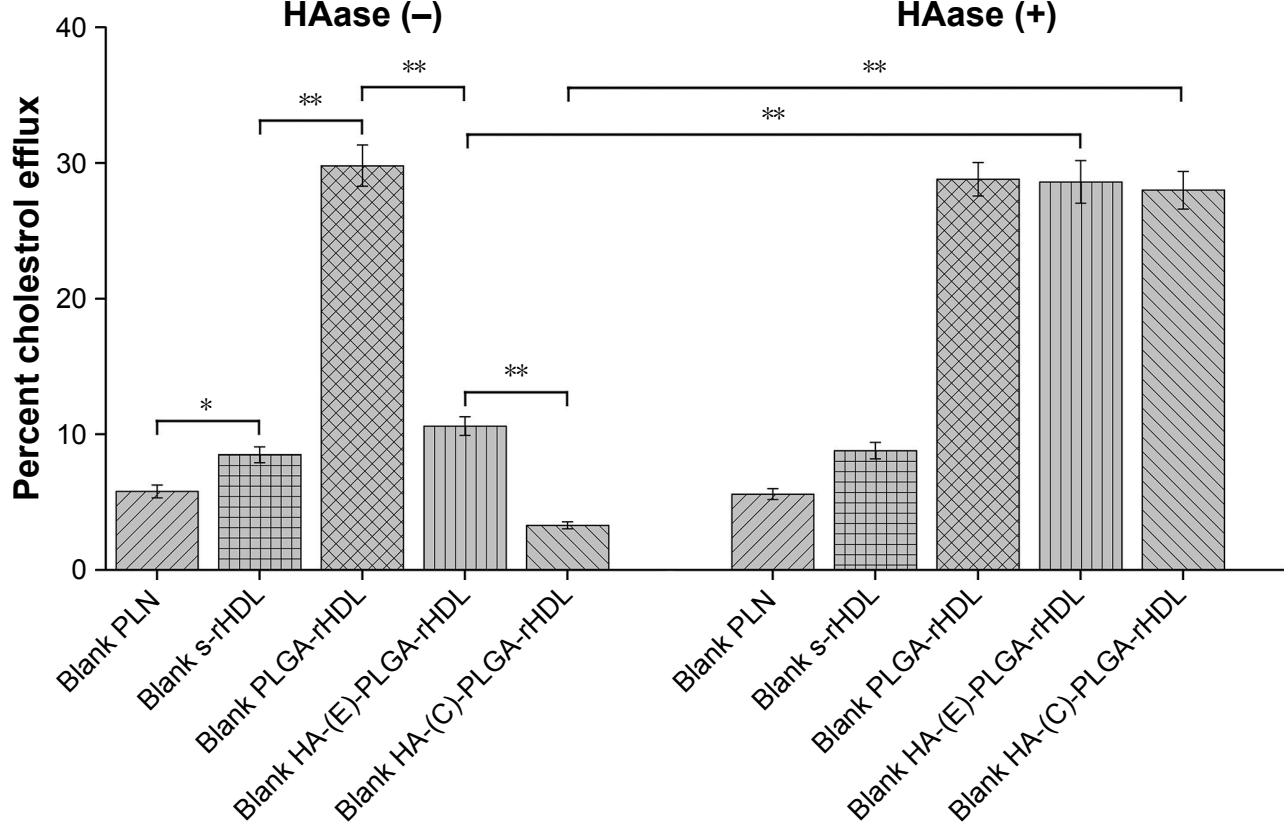

Figure 6 CLSM images (A) of residual NBD-cholesterol in IM $\Phi$ and corresponding percentage of cholesterol efflux (B) from iM $\Phi$ after incubation with blank nanocarriers in the presence (HAase + ) (b) and absence (a) of HAase (HAase -) for $6 \mathrm{~h}$, respectively. $* P<0.05$ and $* * P<0.01$. Images show the blue fluorescence of DAPI, the green fluorescence of NBD-cholesterol, bright field, and the merged fluorescence. Scale bars, $10 \mu \mathrm{m}$. Data are expressed as the mean \pm SD $(n=4)$.

Abbreviations: CLSM, confocal laser scanning microscopy; iMФ, RAW264.7 macrophage cell line; HAase, hyaluronidase; DAPI, 4',6-diamidino-2-phenylindole; SD, standard deviation; NBD-C, 6-[(7-nitro-2, I,3-benzoxadial-4-yl)amino]-cholest-5-en-3-ol; PLN, PLGA-lipid nanoparticle; s-rHDL, spherical rHDL; PLGA, poly (lactic-co-glycolic acid); rHDL, reconstituted high-density lipoprotein; HA, hyaluronan; (E), electrostatically attached; (C), covalently attached.

difference. Moreover, the DiR signal of DiR-HA-(E)-PLGArHDL in aortic trees was obviously stronger than that of DiRPLGA-rHDL $(P<0.01)$, and the strongest fluorescence signal of aortic trees was found in the atherosclerotic rabbit treated with DiR-HA-(C)-PLGA-rHDL. However, the strongest
DiR signals of the liver were detected in DiR-s-rHDL and DiR-PLGA-rHDL groups, and the fluorescence intensities in the liver of free DiR, DiR-PLN, DiR-HA-(E)-PLGArHDL, and DiR-HA-(C)-PLGA-rHDL were decreased in turn (Figure $7 \mathrm{Ab}$ and $\mathrm{Bb}$ ). 

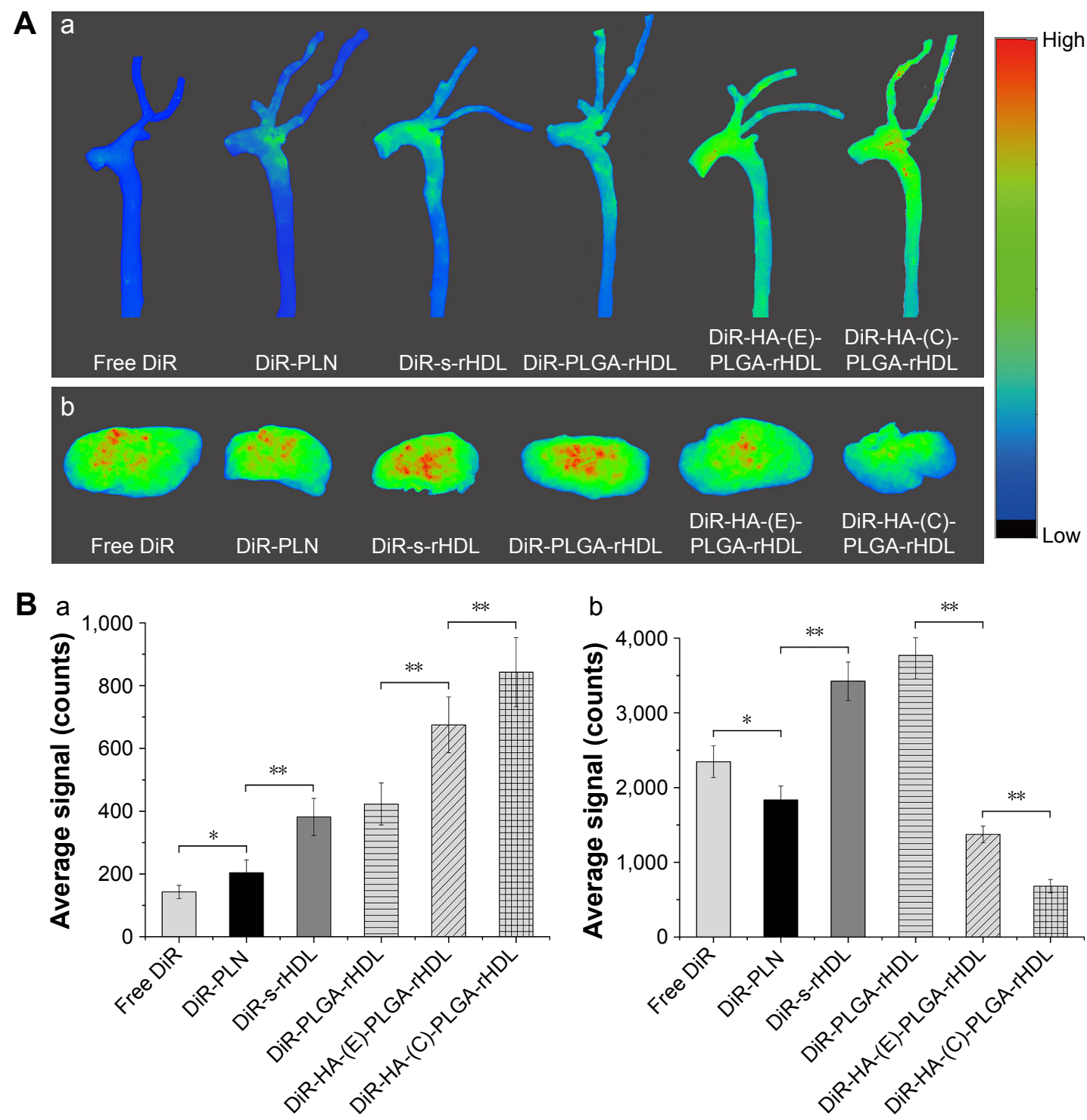

Figure 7 NIRF images $(\mathbf{A})$ and corresponding fluorescence intensity $(\mathbf{B})$ of the aortic trees (a) and livers (b) from atherosclerotic rabbits administrated with free DiR, DiR-PLN, DiR-s-rHDL, DiR-PLGA-rHDL, DiR-HA-(E)-PLGA-rHDL, and DiR-HA-(C)-PLGA-rHDL after 4 h, respectively. $* P<0.05$ and $* * P<0.01$. Data are expressed as the mean \pm SD $(n=6)$.

Abbreviations: NIRF, near-infrared fluorescence; DiR, I,I'-dioctadecyl-3,3,3',3'-tetramethylindotricarbocyanine iodide; PLN, PLGA-lipid nanoparticle; s-rHDL, spherical rHDL; PLGA, poly (lactic-co-glycolic acid); rHDL, reconstituted high-density lipoprotein; HA, hyaluronan; (E), electrostatically attached; (C), covalently attached; $\mathrm{SD}$, standard deviation.

\section{Fluorescence localization of nanoparticles in atherosclerotic aortic roots}

To further investigate the iMФ-targeting efficiency of DiRloaded nanoparticles at a cellular level, CLSM revealed colocalization of each DiR-loaded preparation with an immunofluorescence staining for $\mathrm{iM} \Phi\left(\mathrm{CD} 68^{+}\right)$in aortas of atherosclerotic NZW rabbits. The normal NZW rabbits were treated with DiR-HA-(C)-PLGA-rHDL as the control (Figure 8A). In the aortas of atherosclerotic NZW rabbits, the fluorescence intensity of DiR localized in $\mathrm{iM} \Phi$ ranged from weak to strong in the following order: free DiR, DiR-PLN, DiR-s-rHDL, DiR-PLGA-rHDL, DiR-HA-(E)-PLGA-rHDL, and DiR-HA-(C)-PLGA-rHDL, which was consistent with the results obtained in ex vivo imaging (Figure 8B). Specially, DiR-HA-(C)-PLGA-rHDL displayed 1.56-fold higher fluorescence in iM $\Phi$ than that of DiD-HA-(E)-PLGA-rHDL, and 2.92-fold higher fluorescence than that of DiR-s-rHDL. On the contrary, there was almost no DiR fluorescence located in the $\mathrm{iM} \Phi$ of DiR-HA-(C)-PLGA-rHDL-treated normal NZW rabbits.

\section{In vivo anti-atherogenic efficacies in model animals \\ Oil Red $O$ staining for plaque area analysis}

After Oil Red O staining, atherosclerotic plaque formation in thoracic aorta from each group was evaluated by lesions 


\section{A}

Model

Normal
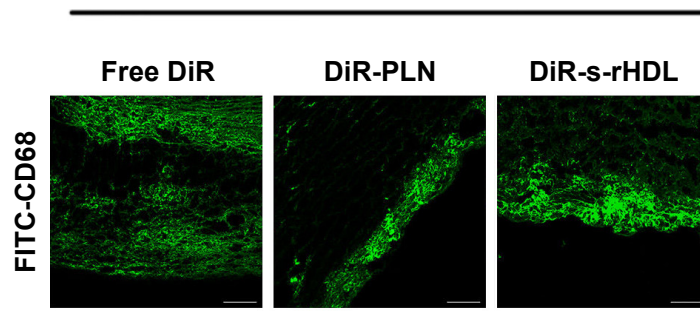

DiR-

DiR-HA-(E)-

DiR-HA-(C)-

DiR-HA-(C)-
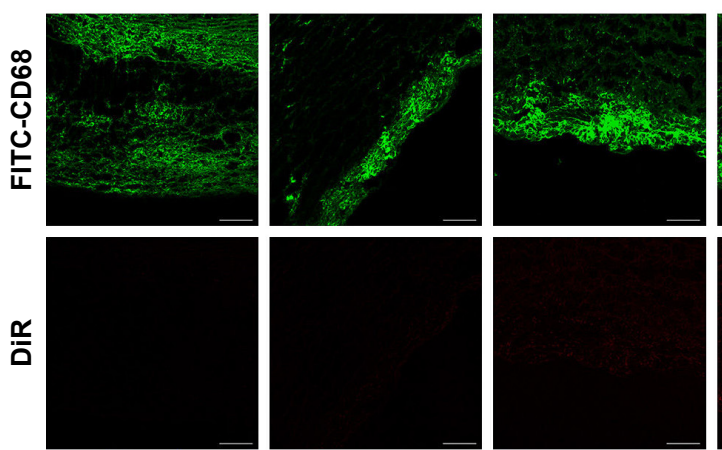

PLGA-rHDL
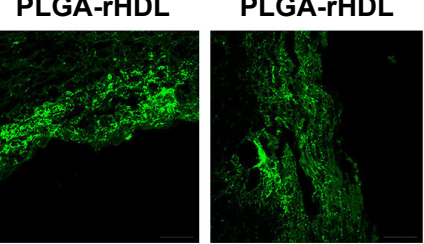

PLGA-rHDL
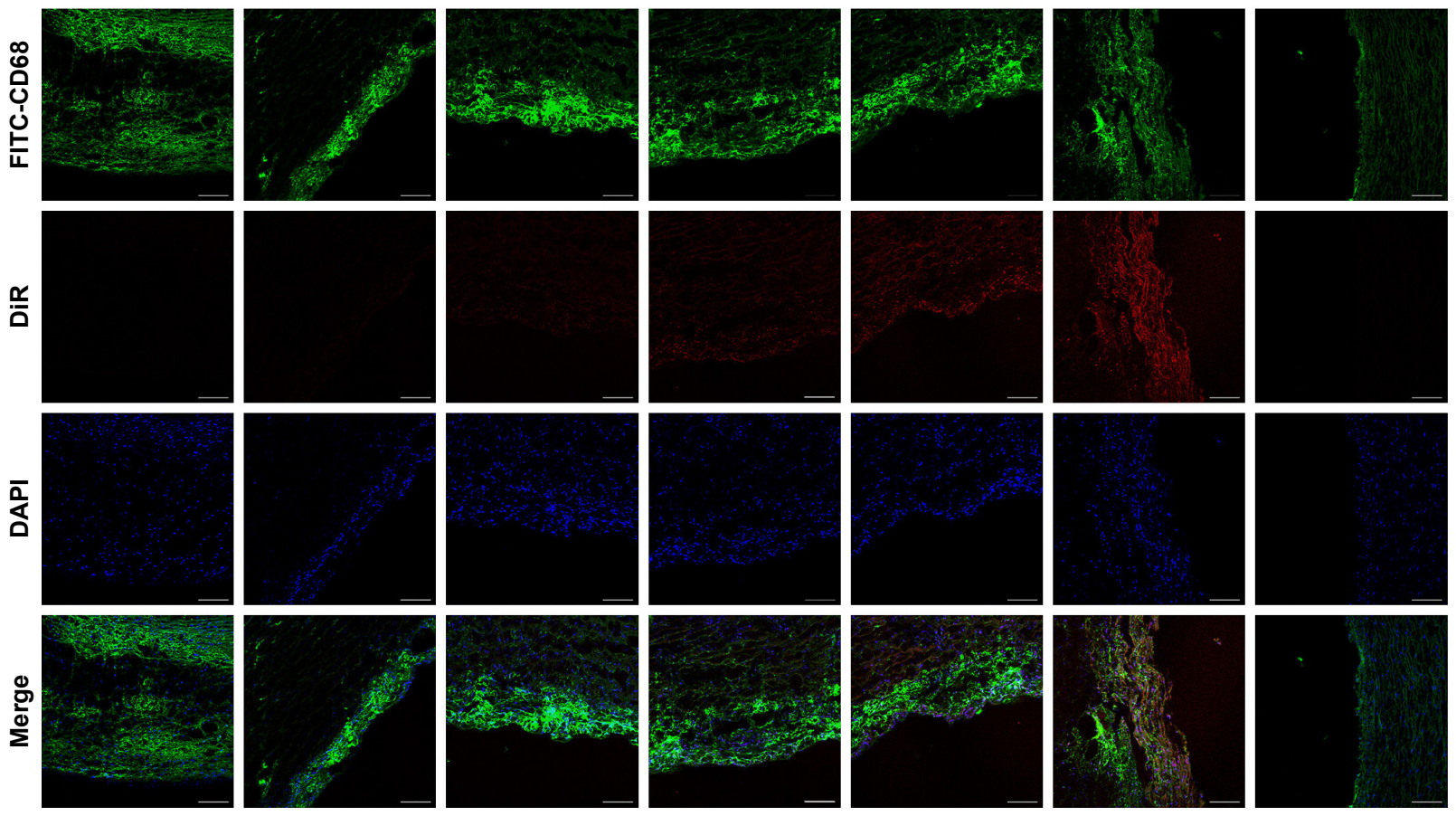

B

Model

\section{Normal}

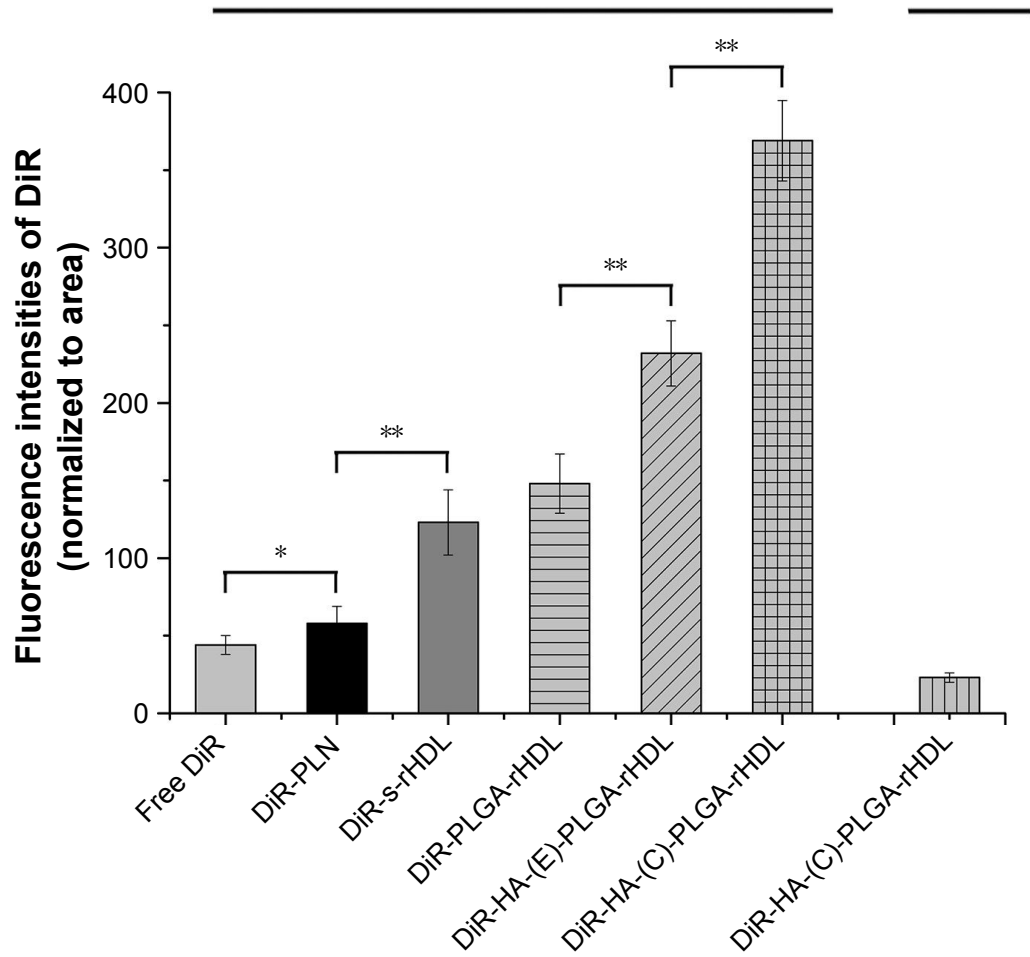

Figure 8 CLSM images (A) and corresponding fluorescence intensity $(\mathbf{B})$ of localization of various DiR-loaded nanocarriers with iM $\Phi$ in aortic trees from atherosclerotic NZW rabbits, and DiR-HA-(C)-PLGA-rHDL with iM $\Phi$ in aortic trees from normal rabbits after 4 h, respectively. $* P<0.05$ and $* * P<0.01$. Images show the green fluorescence of FITC-CD68, the red fluorescence of DiR, the blue fluorescence of DAPI, and the merged fluorescence. Scale bars, $100 \mu \mathrm{m}$. Data are expressed as the mean $\pm S D(n=6)$.

Abbreviations: CLSM, confocal laser scanning microscopy; DiR, I,I'-dioctadecyl-3,3,3',3'-tetramethylindotricarbocyanine iodide; iM Zealand White; HA, hyaluronan; PLGA, poly (lactic-co-glycolic acid); rHDL, reconstituted high-density lipoprotein; FITC, fluorescein isothiocyanate; DAPI, 4',6-diamidino-2phenylindole; SD, standard deviation; PLN, PLGA-lipid nanoparticle; s-rHDL, spherical rHDL; (E), electrostatically attached; (C), covalently attached. 
A

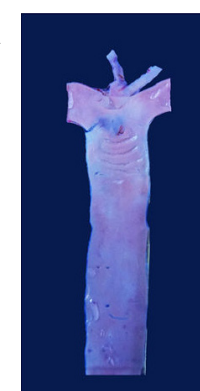

Normal

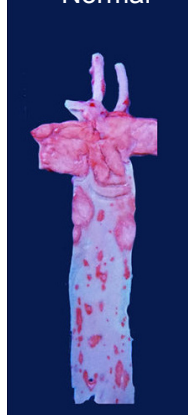

ST-S-rHDL

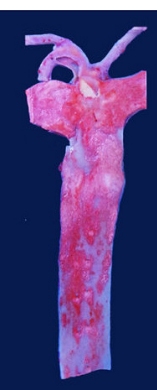

Model

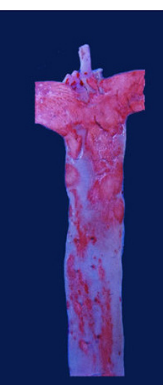

ST-Capsule

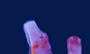

(1)

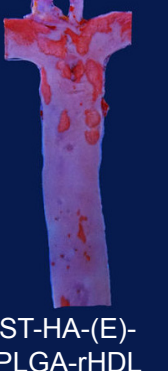

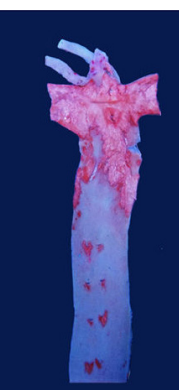

ST-PLN

B

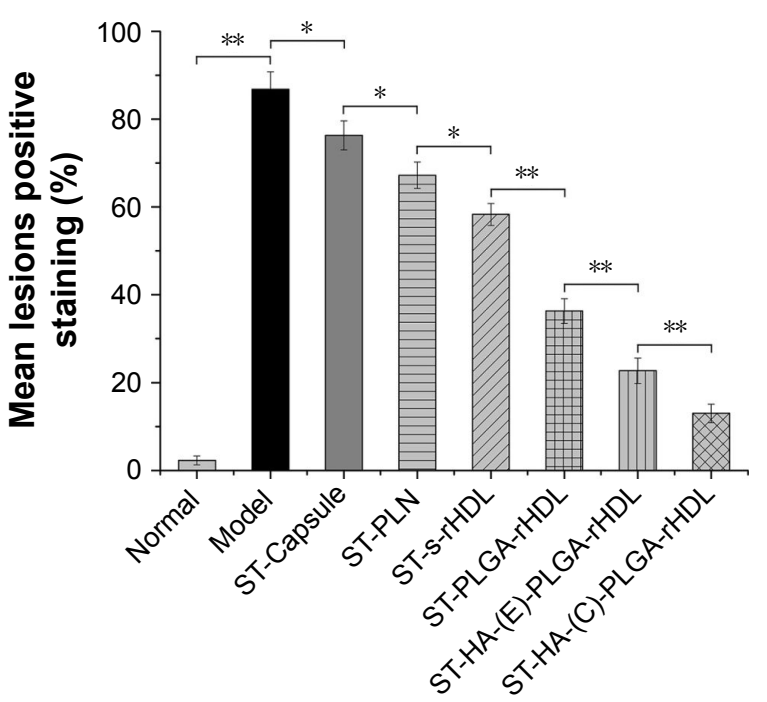

Figure 9 Oil Red O lipid staining (A) and corresponding percentage lesions positive staining (B) of thoracic aorta with different treatments. $* P<0.05$ and $* * P<0.01$. Data are expressed as the mean \pm SD $(n=6)$.

Abbreviations: SD, standard deviation; ST, simvastatin; PLN, PLGA-lipid nanoparticle; s-rHDL, spherical rHDL; PLGA, poly (lactic-co-glycolic acid); rHDL, reconstituted high-density lipoprotein; HA, hyaluronan; $(E)$, electrostatically attached; $(C)$, covalently attached.

positive staining (\%). As shown in Figure 9, model group exhibited significantly larger lesion area $(86.8 \% \pm 4.2 \%)$ compared with other treated groups, whereas the normal group had no atherosclerosis formation. Regarding response in intervened groups, lesions positive staining (\%) of ST-Capsule group was poorer than that of model group $(P<0.05)$; the group treated with ST-PLN exhibited a smaller plaque area compared with ST-Capsule group $(P<0.05)$; the lesions positive staining $(\%)$ from the group treated with ST-s-rHDL was reduced by $21 \%$ compared with ST-PLN group $(P<0.05)$; in contrast with ST-s-rHDL-treated group $(43.8 \% \pm 2.5 \%)$, the lesions positive staining (\%) of ST-PLGA-rHDL-treated group decreased to $31.3 \% \pm 4.3 \%(P<0.01)$; the group treated with ST-HA-(E)-PLGA-rHDL showed apparently smaller staining positive area in contrast with the ST-PLGA-rHDLtreated group $(P<0.01)$, while lesions positive staining $(\%)$ of the group treated with ST-HA-(C)-PLGA-rHDL $(10.9 \% \pm 2.1 \%)$ was attenuated by $38 \%$ compared with STHA-(E)-PLGA-rHDL group $(P<0.01)$.

\section{Immunohistochemistry for $\mathrm{i} M \Phi$ expression}

All groups presented varying positive staining results for iMФ (Figure 10). In contrast with $4.3 \% \pm 1.3 \%$ in the model group, the iM $\Phi$ positive staining (\%) was $80.4 \% \pm 4.3 \%$ in normal group $(P<0.01)$, whereas the $\mathrm{iM} \Phi$ positive staining (\%) was slightly decreased to $71.3 \% \pm 3.2 \%$ in ST-Capsule group compared with that in model group, but without significant difference. As regards the other four groups, iMФ positive staining (\%) of ST-PLN treatment was further decreased compared with ST-Capsule treatment $(P<0.05)$; in the group treated with ST-s-rHDL, the iMФ positive staining (\%) was reduced by $18 \%$ compared with ST-PLN group $(P<0.05)$, and the $\mathrm{iM} \Phi$ positive staining (\%) of ST-PLGA-rHDL was 1.22 times less than that of ST-s-rHDL group $(P<0.05)$; in the group treated with ST-HA-(E)-rHDL, the iM $\Phi$ positive staining (\%) was attenuated to $32.5 \% \pm 2.9 \%$ compared with $43.6 \% \pm 2.5 \%$ in ST-PLGA-rHDL group $(P<0.05)$; the iMФ positive staining (\%) from the group treated with ST-HA(C)-rHDL was 1.71-fold lower than that of ST-HA-(E)-rHDL group $(P<0.01)$.

\section{Gelatinolytic activity assay}

Varied MMP activities among different groups are revealed in Figure 11. It was clear that model group exhibited a much stronger fluorescence signal of MMP than that of normal group $(P<0.01)$. With regard to intervened groups, the green fluorescence intensities were greatly down-regulated except in ST-Capsule group, which showed only a marginal 

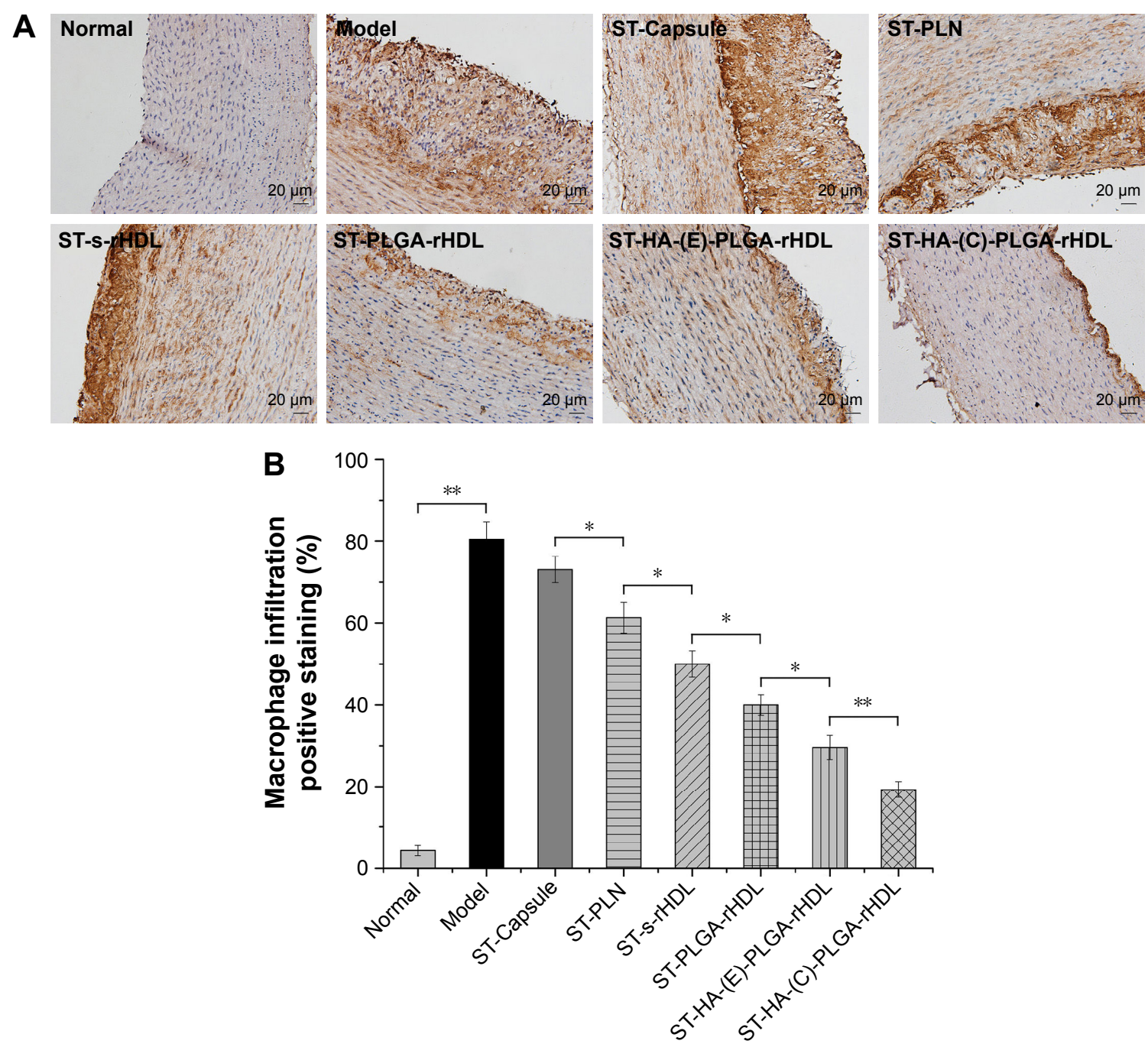

Figure 10 Immunohistochemical staining of iM $\Phi$ infiltration $(\mathbf{A})$ and corresponding percentage iM $\Phi$ positive staining $(\mathbf{B})$ in aortas with different treatments. $* P<0.05$ and $* * P<0.01$. Data are expressed as the mean $\pm S D(n=6)$.

Abbreviations: iM $\Phi$, intimal macrophage; SD, standard deviation; ST, simvastatin; PLN, PLGA-lipid nanoparticle; s-rHDL, spherical rHDL; PLGA, poly (lactic-co-glycolic acid); rHDL, reconstituted high-density lipoprotein; HA, hyaluronan; (E), electrostatically attached; (C), covalently attached.

decrease of fluorescence signal compared with model group. Specifically, ST-PLN group showed weakened green fluorescence intensity compared to ST-Capsule group $(P<0.05)$; less fluorescence signal of MMP occurred in ST-s-rHDL group than that in ST-PLN group $(P<0.05)$; ST-PLGA-rHDL group showed further weakened green fluorescence intensity by $18 \%$ than that of ST-s-rHDL group $(P<0.05)$; compared with ST-PLGA-rHDL group, the fluorescence intensity of ST-HA-(E)-PLGA-rHDL group was significantly decreased $(P<0.05)$, whereas the attenuation effect of ST-HA-(C)PLGA-rHDL on the MMP activities was more powerful than that of ST-HA-(E)-PLGA-rHDL (1.42-fold, $P<0.05$ ).

\section{Discussion}

Increased insight in the role of $\mathrm{MM} \Phi$ in the pathophysiology of atherosclerosis has recently shown promising potential of pharmaceutical interventions against iM $\Phi$ for improving atherosclerosis treatment. ${ }^{3,46,47}$ Despite the inherent atheroprotective properties of rHDL offering superiority over other vehicles for anti-atherogenic therapy, the insufficient specificity of conventional s-rHDL for $\mathrm{iM} \Phi$ and the fact that s-rHDL shows limited cholesterol efflux ability remain challenging issues. These challenges motivated our present research to engineer a novel type of HDL-based nanovector delivering agents into iMФ more efficiently, as well as promoting cholesterol removal from iMФ.

To fulfill this goal, HA-anchored rHDL (HA-(C)-PLGArHDL) was developed in this study. For the synthesis of this nanoparticle, the lipid bilayer encapsulating a polymeric core maintained the spherical structure, and possessed good cholesterol efflux activity aided by apoA-I. Meanwhile, the HAase-responsive HA coated covalently on the surface of 
A
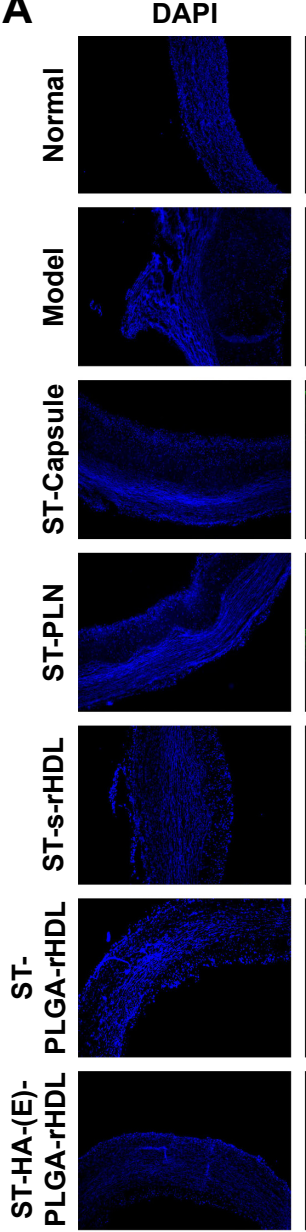

完呈

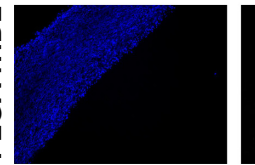

B

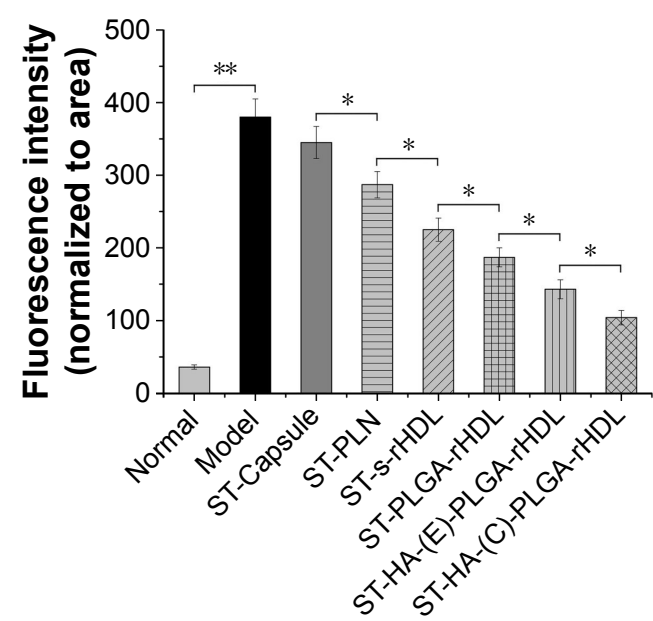

Figure II Immunohistochemical staining of MMP activities (A) and corresponding MMP fluorescence intensities (normalized to area) (B) in aortas with different treatments.

Notes: $* P<0.05$ and $* * P<0.01$. Data are expressed as the mean \pm SD $(n=6) .(A)$ Images captured at magnification $\times 40$.

Abbreviations: MMP, matrix metalloproteinase; SD, standard deviation; DAPI, 4',6diamidino-2-phenylindole; ST, simvastatin; PLN, PLGA-lipid nanoparticle; s-rHDL, spherical rHDL; PLGA, poly (lactic-co-glycolic acid); rHDL, reconstituted high-density lipoprotein; HA, hyaluronan; $(E)$, electrostatically attached; $(C)$, covalently attached. nanoparticles contributed to the decrease of unwanted uptake by liver and the enhanced $\mathrm{i} \Phi \Phi$-targeting efficiency via a multistage-targeting mechanism.

First of all, a modified nanoprecipitation method allowed the preparation of positively charged ST-PLN containing cationic lipid OL. The zeta potential of ST-PLGA-rHDL became slightly lower due to combining with negatively charged apoA-I. After reaction with HA via covalent conjugation or electrostatic absorption, the surface charge reversal of ST-HA-(C)-PLGA-rHDL or ST-HA-(E)-PLGA-rHDL from positive to negative indicated the successful binding of negatively charged polysaccharide chains. As expected, in contrast with ST-HA-(E)-PLGA-rHDL, the lower negative zeta-potential value of ST-HA-(C)-PLGA-rHDL accompanied by a remarkably increased $\mathrm{BE}_{\mathrm{HA}}$ suggested that HA coating via covalent attachment might provide greater shielding coverage for apoA-I than that of electrostatic interaction. DLS measurements revealed the small size of all types of ST-loaded nanoparticles, which could decrease the reticuloendothelial system (RES) capture and facilitate extravasation in atherosclerotic lesions with increased endothelial permeability. ${ }^{48}$ The uniform spherical core-shell shapes of ST-PLN and ST-PLGA-rHDL were confirmed by TEM image, indicative of the white PLGA core wrapped by a lipid bilayer or apoA-I. The electron density of the peripheral region was higher than that of the core. This observation was possibly caused by the negative staining of lipid shell or apoA-I, thus resulting in a gray ring surrounding the PLGA core. ${ }^{49}$ The exclusively hydrophilic corona of ST-HA-(C)-PLGA-rHDL and ST-HA-(E)-PLGA-rHDL could be attributed to the successful decoration of HA on the surface of the ST-PLGA-rHDL, which was analogous to the previous literature. ${ }^{13,33}$ Therefore, the core-multishell structures of ST-HA-(C)-PLGA-rHDL and ST-HA-(E)PLGA-rHDL comprised of the inner PLGA core, medial lipid and apoA-I layer, and the hydrophilic HA coating. Furthermore, the more distinguishable HA layer of ST-HA(C)-PLGA-rHDL compared with ST-HA-(E)-PLGA-rHDL was assigned to the increased $\mathrm{BE}_{\mathrm{HA}}$.

From in vitro release tests, it was revealed that ST from ST-PLN, ST-PLGA-rHDL, ST-HA-(E)-PLGA-rHDL, and ST-HA-(C)-PLGA-rHDL displayed markedly sustainedreleasing rates in the absence of HAase. The lipid bilayer acting as a molecular fence strongly confined the diffusion of drugs from hydrophobic PLGA core, and apoA-I embedded in lipid layer as another hindrance retarded the release of drugs from ST-PLGA-rHDL in contrast with ST-PLN. Moreover, hydrophilic HA molecules on the surface of 
ST-HA-(E)-PLGA-rHDL further prevented the leaching out of drugs, which was consistent with the report that HA would form a hydrophilic matrix around the nanoparticles to restrict the drug release, ${ }^{13}$ while slower diffusion of the drugs was found in ST-HA-(C)-PLGA-rHDL compared with that in ST-HA-(E)-PLGA-rHDL, likely because of greater HA surface coverage. It could also be seen that there was no notable difference in release behaviors of ST-PLGA-rHDL between HAase and no HAase treatment, similar to ST-PLN, whereas enzymatic treatment removed the HA coating of STHA-(C)-PLGA-rHDL and ST-HA-(E)-PLGA-rHDL, resulting in a higher release, similar to that from ST-PLGA-rHDL. This finding implied that both ST-HA-(C)-PLGA-rHDL and ST-HA-(E)-PLGA-rHDL were highly susceptible to HAase, and their inner ST-PLGA-rHDL would be prone to exposure upon encountering HAase in vivo, laying a good foundation for subsequent iM $\Phi$-targeting.

Evidence had shown that ox-LDL as the primary source of cholesterol promoted endothelium permeability in the formation of endothelial dysfunction. The overexpression of CD44 receptors in endothelial cells induced by TNF- $\alpha$ was also the key inflammatory process of atherosclerosis. ${ }^{35,36}$ Based on this, an injured endothelium-macrophage co-culture model mimicking in vivo impaired vascular endothelial barrier of atherosclerotic plaque was established by costimulation with TNF- $\alpha$ and ox-LDL, in order to investigate the iM $\Phi$-targeting abilities and multistage-targeting mechanisms of HA-(C)PLGA-rHDL. Under d-flow conditions, ${ }^{21,39}$ the random entering of DiD-PLN, DiD-s-rHDL, and DiD-PLGA-rHDL into the lower compartment was only based on a simple passive targeting via the enhanced permeability effect of injured HUVECs. The higher intracellular uptake of DiDs-rHDL by iM $\Phi$ than that of DiD-PLN might be associated with apoA-I, through interactions with SR-BI on the surface of $\mathrm{iM} \Phi$. Moreover, the lipid bilayer conducive to the fusion with cell membrane possibly allowed the slightly increased cellular uptake of DiD-PLGA-rHDL as compared to DiD-srHDL. However, the intracellular accumulation of these three types of nanocarriers was far below that of DiD-HA-(E)PLGA-rHDL, and the highest cellular uptake was found in DiD-HA-(C)-PLGA-rHDL. The likely explanation was that HA electrostatically absorbed on DiD-HA-(E)-PLGA-rHDL selectively guided the nanoparticles to CD44-overexpressing damaged region of HUVEC monolayer with enhanced permeability. While, in contrast with DiD-HA-(E)-PLGArHDL, HA chains covalently bonded to DiD-HA-(C)-PLGArHDL with more density and better stability might further provide boosted access to CD44 so as to withstand the drag of d-flow and increase the entry of nanoparticles into the lower compartment, thereby achieving the highest accumulation in iM $\Phi$. This phenomenon demonstrated the superiority of HA modification through covalent bonding for delivering drug into iM $\Phi$ compared to electrostatic adsorption.

Furthermore, the following experiments revealed the multistage-targeting mechanism of HA-(C)-PLGA-rHDL. In the competition assay performed by pretreating the HUVECs with excess free HA to block all the CD44 receptors, a highly diminished cellular uptake was observed both for DiD-HA-(C)-PLGA-rHDL (78\% reduction) and for DiD-HA-(E)-PLGA-rHDL (60\% reduction). This observation suggested that CD44-mediated pathway based upon the active targeting ability of HA would largely contribute to the enhanced uptake of DiD-HA-(C)-PLGA-rHDL and DiD-HA-(E)-PLGA-rHDL by iMФ; moreover, limited cellular localization of DiD-HA-(C)-PLGA-rHDL was shown in iM $\Phi$ after preincubation with BLT-1 for inhibiting SR-BI receptors, demonstrating that the uptake efficiency of DiDHA-(C)-PLGA-rHDL by iM $\Phi$ was also dependent on the SRBI-mediated endocytosis. Similar effects of SR-BI inhibitor occurred on DiD-HA-(E)-PLGA-rHDL, DiD-PLGA-rHDL, and DiD-s-rHDL; it is worth mentioning that the more remarkable reduction in $\mathrm{iM} \Phi$ uptake of DiD-HA-(C)-PLGArHDL was presented in the CD44 competition assay $(78 \%$ reduction), in comparison to the case of suppressing SR-BI receptors (50\% reduction). This meant CD44-mediated pathway was the dominant stage during the multistage-targeting process for delivery of nanotherapeutics specifically to iMФ; additionally, a 34\% decrease in cellular uptake of DiD-HA(E)-PLGA-rHDL without HAase treatment compared to those treated with HAase was observed, suggesting that the HA chains via electrostatic adsorption could impede the interaction between apoA-I and SR-BI receptors, whereas a more dramatic diminution of $58 \%$ in $\mathrm{iM} \Phi$ uptake of the HAaseuntreated DiD-HA-(C)-PLGA-rHDL indicated that the HA shell via covalent binding could act as a more effective shield against the recognition of apoA-I by SR-BI. As such, it was reasonably speculated that DiD-HA-(C)-PLGA-rHDL might be capable of decreasing the non-targeted uptake by liver, prior to entering into atherosclerotic plaque in vivo. Taken together, the targeting process of HA-(C)-PLGA-rHDL may have the following stages: in the first stage, HA coating on the nanoparticles reduces the unwanted uptake by liver as well as facilitating the accumulation of HA-(C)-PLGArHDL in injured endothelium with atherosclerotic plaque by strongly adhering to the overexpressed CD44 receptors; in the second stage, HA-(C)-PLGA-rHDL travels through the 
injured endothelial layer with enhanced permeability effect into the subendothelial space, and exhibits HAase-responsive exposure of the inner PLGA-rHDL; in the third stage, naked PLGA-rHDL targets into $\mathrm{iM} \Phi$ via SR-BI-mediated endocytosis, ultimately.

A critical anti-atherogenic bioactivity of HDL is considered to be its ability to induce cholesterol efflux from iMФ. To examine this essential bioactive property of our HDL-based nanoparticles, cholesterol efflux-time curves of each blank nanocarrier pretreated with or without HAase in iMФ were respectively graphed. Cellular cholesterol was removed at equilibrium after $6 \mathrm{~h}$. It is generally accepted that pathways responsible for cholesterol efflux involve passive diffusion and apoA-I-mediated transports linked to specific efflux membrane proteins, such as SR-BI and ATP-binding cassette transporter A1 and G1 (ABCA1, ABCG1). ${ }^{16,22}$ At the cholesterol-effluxing equilibrium state of nanocarriers without HAase treatment, PLN displayed little ability to efflux cholesterol from $\mathrm{iM} \Phi$, which might only depend upon the inefficient passive diffusion, driven by the cholesterol concentration gradient between donor and acceptor. While the higher level of cholesterol efflux induced by s-rHDL compared to PLN might be attributable to the active pathway of apoA-I-mediated efflux, ${ }^{22}$ via the more excellent apoA-I-mediated active efflux compared to s-rHDL, PLGA-rHDL functionalized with a polymer core without additional cholesterol, and lipid bilayer having the larger available space for cholesterol inclusion caused greatest cholesterol efflux; however, due to the steric hindrance of HA hydrophilic chains hampering the interaction of apoA-I with efflux membrane proteins, cells treated with HA-(E)-PLGA-rHDL exhibited little cholesterol efflux; ${ }^{26}$ in particular, the lesser amount of cholesterol efflux mediated by HA-(C)-PLGA-rHDL than that of HA-(E)-PLGA-rHDL indirectly reaffirmed the greater HA surface coverage of DiD-HA-(C)-PLGA-rHDL for masking the function of apoA-I more effectively. Intriguingly, as compared to their respective HAase-untreated nanoparticles, HA-(C)-PLGArHDL pretreated with HAase generated notably increased cholesterol efflux nearly equal to the efflux efficiency of PLGA-rHDL. This finding supported the expectation that this HAase-responsive HA-(C)-PLGA-rHDL could still possess attractive cholesterol efflux capability after the degradation of outermost HA chains by HAase in vivo, even apparently better than that of conventional s-rHDL (2.43-fold).

Unsurprisingly, ex vivo imaging confirmed the highest $\mathrm{DiR}$ presence in the aortic tree and the lowest signal intensity in the liver from atherosclerotic rabbits injected with DiR-HA-(C)-PLGA-rHDL, which could be explained by mainly two aspects: (i) the protective HA coating covalently immobilized on DiR-HA-(C)-PLGA-rHDL possessed "stealth" characters similar to hydrophilic material polyethylene glycol, ${ }^{50}$ and maximized the shielding for apoA-I function so as to avoid the undesired internalization by liver in circulation; and (ii) with the aid of the high affinity of HA toward CD44 receptors up-regulated on damaged endothelial cells, DiR-HA-(C)-PLGA-rHDL was capable of vanquishing the drag of d-flow at atherosclerotic lesions, and various biological barriers for targeting iM $\Phi$ via the multistagetargeting mechanism. Most importantly, the strongest DiR signal specifically colocalized with CD68-stained iM $\Phi$ further corroborated the excellent targeting performance of DiR-HA-(C)-PLGA-rHDL on $\mathrm{iM} \Phi$ at a cellular level; in accord with the observations from in vitro $\mathrm{iM} \Phi$-targeting experiments, insufficient HA surface coverage for masking apoA-I and the inferior stability of HA electrostatically attached to DiR-HA-(E)-PLGA-rHDL might help explain the higher distribution in liver and lower accumulation of DiR-HA-(E)-PLGA-rHDL in the iM $\Phi$ than that of DiR-HA(C)-PLGA-rHDL; compared with other nanocarriers, a great deal of DiR-PLGA-rHDL and DiR-s-rHDL were phagocytosed by liver via SR-BI receptor-mediated pathway prior to being delivered into the target, thus resulting in the largest accumulation in liver and decreasing the distribution in $\mathrm{iM} \Phi$, which was analogous to the results in our previous study; besides, probably ascribed to the lipid bilayer conducive to internalization by cells, DiR-PLGA-rHDL exhibited slightly better iM $\Phi$-targeting effect than DiR-s-rHDL; the inefficient passive targeting under the interference of d-flow and higher uptake by RES in circulation might presumably account for the weakest signal intensity of DiR-PLN in iM $\Phi$; because of lack of specificity, free DiR almost had no deposition in the iM $\Phi$ of atherosclerotic lesions. All these data of DiR-HA(C)-PLGA-rHDL were encouraging and paved the path for in vivo efficacy studies. Additionally, these results were all in agreement with that from in vitro cell experiments conducted in the established injured endothelium-macrophage co-culture dynamic system, indicating the potentiality of the co-culture dynamic model in predicting the in vivo iMФtargeting ability and mechanisms of nanocarriers.

To verify the feasibility of ST-HA-(C)-PLGA-rHDL for anti-atherogenic therapy in vivo, intervention treatment was undertaken in model rabbits, and several universally acknowledged indices were chosen as evaluation criterions. In this experiment, ST-solution was replaced by commercial ST-Capsule as the control preparation because of the 
serious adverse effects resulting from long-term intravenous administration of ST formulated in the $40 \%$ dimethyl sulfoxide solution. Compared with untreated model group, the obviously reduced plaque area of commercial ST-Capsule mainly resulted from the classical lipid-lowering effects of stains on hepatocytes. Nevertheless, no noticeable improvement was observed in other anti-atherogenic indices including $\mathrm{iM} \Phi$ infiltration and gelatinolytic activity relative to the pleiotropic benefits of statins, which were greatly hindered by the poor bioavailability of ST-Capsule and nonspecificity for iM $\Phi .{ }^{31}$ As for nano-sized preparations, the atheroprotective efficacies were positively correlated with the intracellular accumulation of statins in $\mathrm{iM} \Phi$. All indices of nano-sized preparations were significantly improved in contrast to ST-Capsule group, probably owing to the exploitation of pleiotropic effects of statins. The accumulated stains in iM $\Phi$ could significantly depress the iM $\Phi$ infiltration in atherosclerotic plaque and greatly lower the MMP activities, which was also supported by many studies where ST acted as immunomodulating agent for anti-atherosclerotic therapy through inhibiting the intracellular mevalonate pathway. ${ }^{51,52}$ In addition, in consideration of the only slightly better iMФ-targeting effect of PLGA-rHDL compared with s-rHDL, the obviously enhanced anti-atherosclerotic potency of ST-PLGA-rHDL compared to that of ST-s-rHDL may be mainly associated with the stronger cholesterol efflux property of blank PLGArHDL. Exhilaratingly, ST-HA-(C)-PLGA-rHDL possessed the most potent atheroprotective activities, and this dramatic therapeutic improvement could be firstly due to the capability of ST-HA-(C)-PLGA-rHDL to decrease the liver uptake under the better shielding effect of covalent HA coating than that of ST-HA-(E)-PLGA-rHDL with electrostatic HA binding, and conquer multiple barriers for delivering statins into iMФ more efficiently via a multistage-targeting strategy, which was also verified by in vitro iM $\Phi$-targeting mechanism investigation, ex vivo imaging, and fluorescence localization. Secondly, the anti-atherogenic benefits would also be accompanied by the excellent function of blank HA(C)-PLGA-rHDL that facilitates cellular cholesterol efflux after the degradation of HA coating by HAase, which was revealed by results from cellular cholesterol efflux assay. Last but not the least, these synergistic anti-atherogenic effects of apoA-I, such as anti-oxidation and anti-inflammatory, might further reinforce the efficacies of HA-(C)-PLGA-rHDL. ${ }^{53}$

Collectively, the current study indicated that this HAaseresponsive HA-(C)-PLGA-rHDL possessing attractive ability to overcome the physiological barriers of atherosclerotic plaques for efficient iM $\Phi$-targeted drug delivery, and simultaneously facilitate cellular cholesterol removal, could be exploited as a promising drug delivery system for remarkably suppressing the advancement of atherosclerosis.

\section{Conclusion}

The HDL-mimetic nanocarrier developed in the present study are not only capable of specifically delivering anti-atherogenic agents into $\mathrm{iM} \Phi$ via a multistage way, but also promoting cholesterol removal from iMФ. HA-(C)-PLGA-rHDL, the biologically inspired nanoparticle with a particle size of $138 \mathrm{~nm}$ constructed in this study, mainly mimicked the bioactivity of native HDL. This study showed that HA-(C)-PLGA-rHDL greatly decreased the off-target uptake by liver via SR-BI, remarkably improved iM $\Phi$ targeting via a multistage-targeting mechanism, and achieved excellent cellular cholesterol efflux. In vivo efficacy studies eventually highlighted the great possibilities of HA-(C)-PLGA-rHDL as an iMФ-targeted nanocarrier with excellent cholesterol efflux capacity for treating atherosclerosis effectively. These findings not only give new insights to design high-performance HDL-based nanovehicles but also provide strong proof for the efficient anti-atherogenic therapy via the $\mathrm{iM} \Phi$-targeted drug delivery strategy.

\section{Acknowledgments}

The project described was financially supported by the National Natural Science Foundation of China (No 81273466), Jiangsu Province Ordinary College and University Innovative Research Programs (No KYLX_0614), and the Priority Academic Program Development of Jiangsu Higher Education Institutions. The authors also acknowledge Atherosclerosis Research Centre (Nanjing Medical University, Nanjing, People's Republic of China) for the kind gift of HUVEC line and murine macrophage cell line, and the technical support from KeyGEN BioTECH (Nanjing, Jiangsu, People's Republic of China).

\section{Disclosure}

The authors report no conflicts of interest in this work.

\section{References}

1. Weber $\mathrm{C}$, Noels $\mathrm{H}$. Atherosclerosis: current pathogenesis and therapeutic options. Nat Med. 2011;17(11):1410-1422.

2. Wu XH, Zhang H, Wu ZG. Macrophage and atherosclerosis. $A d v$ Cardiovasc Dis. 2005;26(3):302-306.

3. Tabas I. Macrophage death and defective infammation resolution in atherosclerosis. Nat Rev Immunol. 2010;10(1):36-46.

4. Moore KJ, Sheedy FJ, Fisher EA. Macrophages in atherosclerosis: a dynamic balance. Nat Rev Immunol. 2013;13(10):709-721.

5. You DG, Saravanakumar G, Son S, et al. Dextran sulfate-coated superparamagnetic iron oxide nanoparticles as a contrast agent for atherosclerosis imaging. Carbohydr Polym. 2014;101:1225-1233. 
6. Nie S, Zhang J, Martinez-Zaguilan R, et al. Detection of atherosclerotic lesions and intimal macrophages using CD36-targeted nanovesicles. $J$ Control Release. 2015;220(Pt A):61-70.

7. Damiano MG, Mutharasan RK, Tripathy S, Mcmahon KM, Thaxton CS. Templated high density lipoprotein nanoparticles as potential therapies and for molecular delivery. Adv Drug Deliv Rev. 2013; 65(5):649-662.

8. Fisher EA, Feig JE, Hewing B, Hazen SL, Smith JD. High-density lipoprotein function, dysfunction, and reverse cholesterol transport Arterioscler Thromb Vasc Biol. 2012;32(12):2813-2820.

9. Khera AV, Cuchel M, de la Llera-Moya M, et al. Cholesterol efflux capacity, high-density lipoprotein function, and atherosclerosis. $N E n g l$ J Med. 2011;364(2):127-135.

10. Zhang W, He H, Liu J, et al. Pharmacokinetics and atherosclerotic lesions targeting effects of tanshinone IIA discoidal and spherical biomimetic high density lipoproteins. Biomaterials. 2013;34(1):306-319.

11. Ji A, Wroblewski JM, Cai L, de Beer MC, Webb NR, van der Westhuyzen DR. Nascent HDL formation in hepatocytes and role of ABCA1, ABCG1, and SR-BI. J Lipid Res. 2012;53(3):446-455.

12. Rui M, Guo W, Ding Q, Wei X, Xu J, Xu Y. Recombinant high-density lipoprotein nanoparticles containing gadolinium-labeled cholesterol for morphologic and functional magnetic resonance imaging of the liver. Int J Nanomedicine. 2012;7:3751-3768.

13. Liu L, He H, Zhang M, Zhang S, Zhang W, Liu J. Hyaluronic aciddecorated reconstituted high density lipoprotein targeting atherosclerotic lesions. Biomaterials. 2014;35(27):8002-8014.

14. Slevin M, Krupinski J, Gaffney J, et al. Hyaluronan-mediated angiogenesis in vascular disease: uncovering RHAMM and CD44 receptor signaling pathways. Matrix Biol. 2007;26(1):58-68.

15. Poon Z, Lee JB, Morton SW, Hammond PT. Controlling in vivo stability and biodistribution in electrostatically assembled nanoparticles for systemic delivery. Nano Lett. 2011;11(5):2096-2103.

16. Hoang A, Drew BG, Low H, et al. Mechanism of cholesterol efflux in humans after infusion of reconstituted high-density lipoprotein. Eur Heart J. 2012;33(5):657-665.

17. He H, Zhang M, Liu L, Zhang S, Liu J, Zhang W. Suppression of remodeling behaviors with arachidonic acid modification for enhanced in vivo antiatherogenic efficacies of lovastatin-loaded discoidal recombinant high density lipoprotein. Pharm Res. 2015;32(10):3415-3431.

18. Zhang WL, Xiao Y, Liu JP, et al. Structure and remodeling behavior of drug-loaded high density lipoproteins and their atherosclerotic plaque targeting mechanism in foam cell model. Int J Pharm. 2011; 419(1-2):314-321

19. He H, Liu L, Bai H, et al. Arachidonic acid-modified lovastatin discoidal reconstituted high density lipoprotein markedly decreases the drug leakage during the remodeling behaviors induced by lecithin cholesterol acyltransferase. Pharm Res. 2014;31(7):1689-1709.

20. Zhang M, Jia J, Liu J, He H, Liu L. A novel modified paclitaxel-loaded discoidal recombinant high-density lipoproteins: preparation, characterizations and in vivo evaluation. Asian J Pharm Sci. 2013;8(1):11-18.

21. Zeng L, Zampetaki A, Margariti A, et al. Sustained activation of XBP1 splicing leads to endothelial apoptosis and atherosclerosis development in response to disturbed flow. Proc Natl Acad Sci U S A. 2009; 106(20):8326-8331.

22. Phillips MC. Molecular mechanisms of cellular cholesterol efflux. J Biol Chem. 2014;289(35):24020-24029.

23. Lamichhane SP, Arya N, Ojha N, Kohler E, Shastri VP. Glycosaminoglycan-functionalized poly-lactide-co-glycolide nanoparticles: synthesis, characterization, cytocompatibility, and cellular uptake. Int J Nanomedicine. 2015;10:775-789.

24. Wang X, Shi L, Tu Q, et al. Treating cutaneous squamous cell carcinoma using 5-aminolevulinic acid polylactic-co-glycolic acid nanoparticlemediated photodynamic therapy in a mouse model. Int J Nanomedicine. 2015;10:347-355.

25. Marrache S, Dhar S. Biodegradable synthetic high-density lipoprotein nanoparticles for atherosclerosis. Proc Natl Acad Sci U S A. 2013; 110(23):9445-9450.
26. Murphy AJ, Funt S, Gorman D, Tall AR, Wang N. Pegylation of high-density lipoprotein decreases plasma clearance and enhances antiatherogenic activity. Circ Res. 2013;113(1):e1-e9.

27. Bot PT, Pasterkamp G, Goumans MJ, et al. Hyaluronic acid metabolism is increased in unstable plaques. Eur J Clin Invest. 2010;40(9): 818-827.

28. Kim SE, Thanh Thuy TT, Lee JH, et al. Simvastatin inhibits induction of matrix metalloproteinase-9 in rat alveolar macrophages exposed to cigarette smoke extract. Exp Mol Med. 2009;41(4):277-287.

29. Tsuchiya A, Nagotani S, Hayashi T, et al. Macrophage infiltration, lectin-like oxidized-LDL receptor-1, and monocyte chemoattractant protein-1 are reduced by chronic HMG-CoA reductase inhibition. Curr Neurovasc Res. 2007;4(4):268-273.

30. Matsumoto M, Einhaus D, Gold ES, Aderem A. Simvastatin augments lipopolysaccharide-induced proinflammatory responses in macrophages by differential regulation of the c-Fos and c-Jun transcription factors. J Immunol. 2004;172(12):7377-7384.

31. Romana B, Batger M, Prestidge CA, Colombo G, Sonvico F. Expanding the therapeutic potential of statins by means of nanotechnology enabled drug delivery systems. Curr Top Med Chem. 2014;14(9):1182-1193.

32. Zheng $\mathrm{C}$, Zheng $\mathrm{M}$, Gong $\mathrm{P}$, et al. Indocyanine green-loaded biodegradable tumor targeting nanoprobes for in vitro and in vivo imaging. Biomaterials. 2012;33(22):5603-5609.

33. Gan L, Wang J, Zhao Y, et al. Hyaluronan-modified core-shell liponanoparticles targeting CD44-positive retinal pigment epithelium cells via intravitreal injection. Biomaterials. 2013;34(24):5978-5987.

34. Zhu H, Chen H, Zeng X, et al. Co-delivery of chemotherapeutic drugs with vitamin E TPGS by porous PLGA nanoparticles for enhanced chemotherapy against multi-drug resistance. Biomaterials. 2014; 35(7):2391-2400.

35. Chen KS, Chen PN, Hsieh YS, Lin CY, Lee YH, Chu SC. Capsaicin protects endothelial cells and macrophage against oxidized low-density lipoprotein-induced injury by direct antioxidant action. Chem Biol Interact. 2015;228:35-45.

36. Gutiérrez G, Mendoza C, Zapata E, et al. Dehydroepiandrosterone inhibits the TNF-alpha-induced inflammatory response in human umbilical vein endothelial cells. Atherosclerosis. 2007;190(1):90-99.

37. Hood ED, Greineder CF, Dodia C, et al. Antioxidant protection by PECAM-targeted delivery of a novel NADPH-oxidase inhibitor to the endothelium in vitro and in vivo. J Control Release. 2012;163(2): $161-169$.

38. Croes M, Oner FC, Kruyt MC, et al. Proinflammatory mediators enhance the osteogenesis of human mesenchymal stem cells after lineage commitment. PLoS One. 2015;10(7):e0132781.

39. Martin D, Li Y, Yang J, et al. Unspliced X-box-binding protein 1 (XBP1) protects endothelial cells from oxidative stress through interaction with histone deacetylase 3. J Biol Chem . 2014;289(44):30625-30634.

40. Yu M, Romer KA, Nieland TJ, et al. Exoplasmic cysteine Cys 384 of the HDL receptor SR-BI is critical for its sensitivity to a small-molecule inhibitor and normal lipid transport activity. Proc Natl Acad Sci USA. 2011;108(30):12243-12248.

41. Chen CY, Shyue SK, Ching LC, et al. Wogonin promotes cholesterol efflux by increasing protein phosphatase 2B-dependent dephosphorylation at ATP-binding cassette transporter-A1 in macrophages. J Nutr Biochem. 2011;22(11):1015-1021.

42. Lorenz M, Fechner M, Kalkowski J, et al. Effects of lycopene on the initial state of atherosclerosis in New Zealand White (NZW) rabbits. PLoS One. 2012;7(1):e30808.

43. Feig JE, Vengrenyuk Y, Reiser V, et al. Regression of atherosclerosis is characterized by broad changes in the plaque macrophage transcriptome. PLoS One. 2012;7(6):e39790.

44. Juengel E, Krueger G, Rutz J, et al. Renal cell carcinoma alters endothelial receptor expression responsible for leukocyte adhesion. Oncotarget. 2016;7(15):20410-20424.

45. Lobatto ME, Fuster V, Fayad ZA, Mulder WJ. Perspectives and opportunities for nanomedicine in the management of atherosclerosis. Nat Rev Drug Discov. 2011;10(11):835-852. 
46. Moore KJ, Tabas I. Macrophages in the pathogenesis of atherosclerosis. Cell. 2011;145(3):341-355.

47. Williams HJ, Fisher EA, Greaves DR. Macrophage differentiation and function in atherosclerosis: opportunities for therapeutic intervention? J Innate Immun. 2012;4(5-6):498-508.

48. Zhang C, Qu G, Sun Y, et al. Pharmacokinetics, biodistribution, efficacy and safety of N-octyl-O-sulfate chitosan micelles loaded with paclitaxel. Biomaterials. 2008;29(9):1233-1241.

49. Zhang L, Chan JM, Gu FX, et al. Self-assembled lipid-polymer hybrid nanoparticles: a robust drug delivery platform. ACS Nano. 2008;2(8): 1696-1702.

50. Dosio F, Arpicco S, Stella B, Fattal E. Hyaluronic acid for anticancer drug and nucleic acid delivery. Adv Drug Deliv Rev. 2016;97:204-236.
51. Duivenvoorden R, Tang J, Cormode DP, et al. A statin-loaded reconstituted high-density lipoprotein nanoparticle inhibits atherosclerotic plaque inflammation. Nat Commun. 2014;5:3065.

52. Croons V, De Meyer I, Houten SM, et al. Effect of statins on the viability of macrophages and smooth muscle cells. J Cardiovasc Pharmacol. 2010;55(3):269-275.

53. Pedersen TX, Bro S, Andersen MH, et al. Effect of treatment with human apolipoprotein A-I on atherosclerosis in uremic apolipoprotein-E deficient mice. Atherosclerosis. 2009;202(2):372-381. 


\section{Supplementary materials Cytotoxicity assays}

The cytotoxicities of different blank nanocarriers were evaluated using an MTT assay. In detail, RAW264.7 macrophage cell line and human umbilical vein endothelial cells (kindly gifted by Atherosclerosis Research Centre, Nanjing Medical University, Nanjing, People's Republic of China) were seeded in 96-well plates at a density of 10,000 cells/well and cultured in M199 containing 20\% heat-inactivated fetal bovine serum, $100 \mathrm{U} / \mathrm{mL}$ penicillin $\mathrm{G}$, $100 \mathrm{mg} / \mathrm{mL}$ streptomycin supplemented with $25 \mu \mathrm{g} / \mathrm{mL}$ endothelial cell growth supplement, $10 \mathrm{mM}$ L-glutamine, and $0.75 \mathrm{U} / \mathrm{mL}$ heparin (Thermo Fisher Scientific, Waltham,
MA, USA) for $24 \mathrm{~h}$ under $5 \% \mathrm{CO}_{2}$ at $37^{\circ} \mathrm{C}$, respectively. The medium was then replaced with $200 \mu \mathrm{L}$ fresh medium containing blank poly (lactic-co-glycolic acid) (PLGA)lipid nanoparticle, spherical reconstituted high-density lipoprotein (rHDL), PLGA-rHDL, hyaluronan (HA) electrostatically attached to PLGA-rHDL, and HA covalently attached to PLGA-rHDL at various concentrations, respectively. After incubation for $24 \mathrm{~h}$, MTT $(5 \mathrm{mg} / \mathrm{mL}$ in $\mathrm{pH} 7.4$ phosphate-buffered saline) was added into each well, and the two different kinds of cell lines were further incubated with the mixture for $4 \mathrm{~h}$. The absorbance of each well was measured at $570 \mathrm{~nm}$ on a microplate reader following the addition of dimethyl sulfoxide.
A

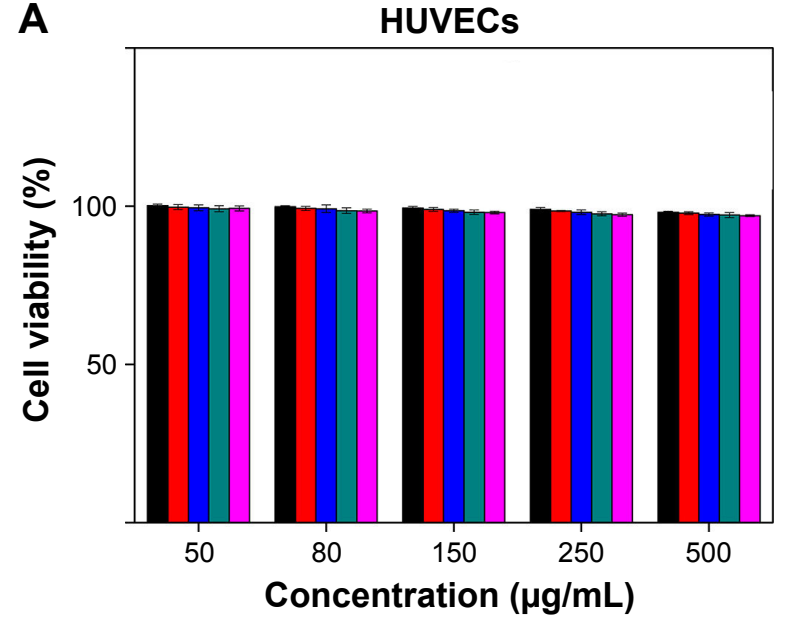

B

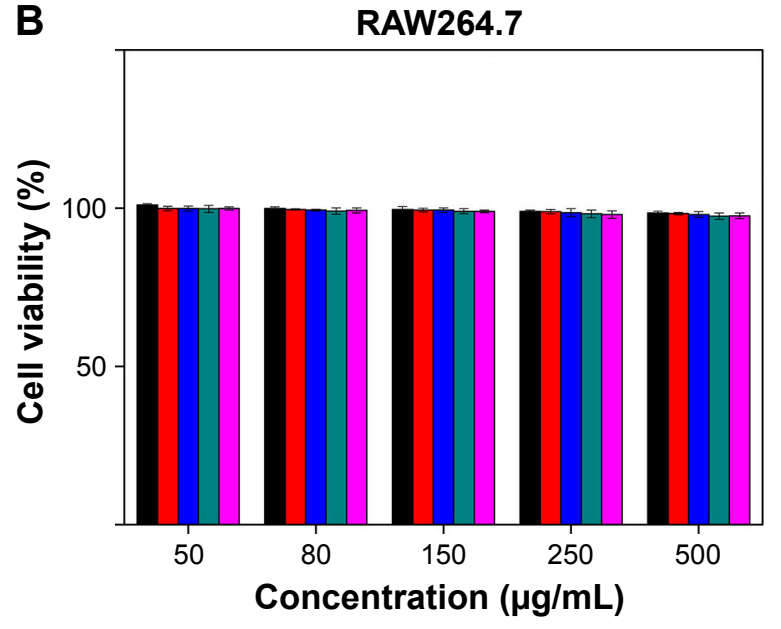

Blank PLN $\square$ Blank s-rHDL $\square$ Blank PLGA-rHDL $\square$ Blank HA-(E)-PLGA-rHDL $\square$ Blank HA-(C)-PLGA-rHDL

Figure SI Cytotoxicity of various blank nanocarriers against HUVECs (A) and iMФ (B) with concentrations ranging from 20 to $500 \mu g / \mathrm{mL}$. Data are expressed as the mean \pm SD $(n=3)$.

Abbreviations: HUVECs, human umbilical vein endothelial cells; iMФ, RAW264.7 macrophage cell line; SD, standard deviation; PLN, PLGA-lipid nanoparticle; s-rHDL, spherical rHDL; PLGA, poly (lactic-co-glycolic acid); rHDL, reconstituted high-density lipoprotein; HA, hyaluronan; (E), electrostatically attached; (C), covalently attached. 

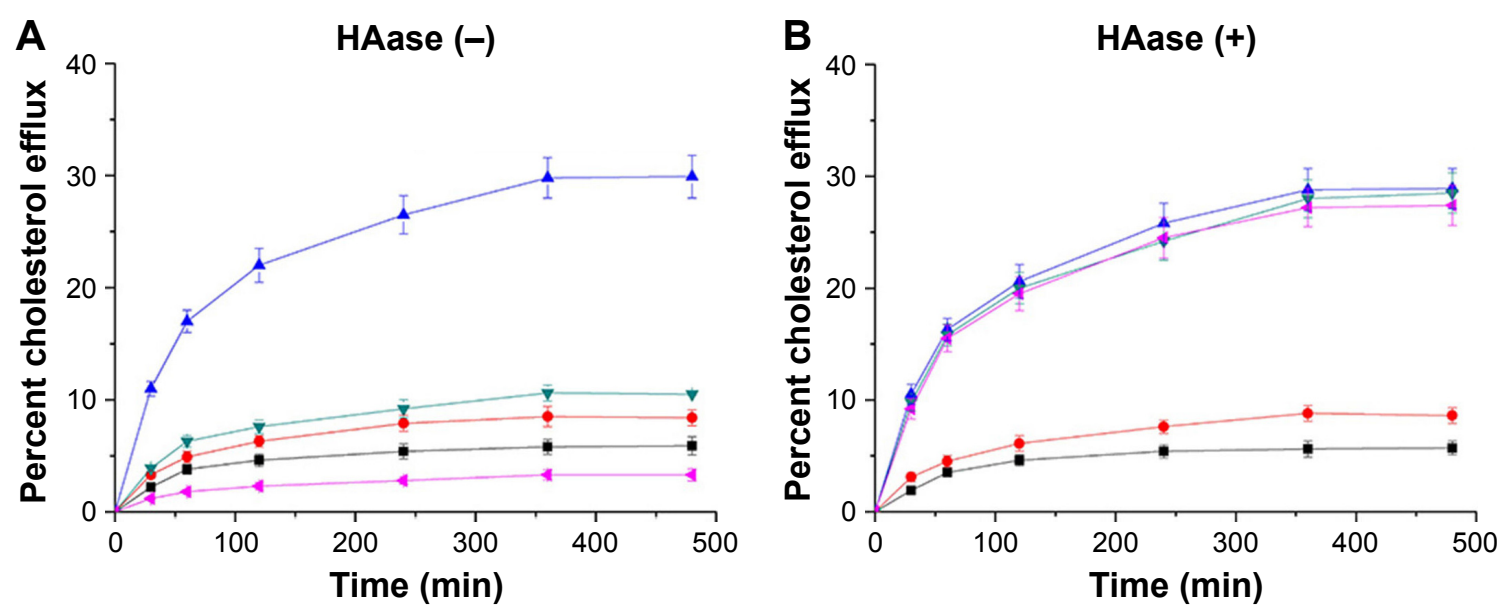

Blank PLN $\rightarrow$ Blank s-rHDL $\quad$ - Blank PLGA-rHDL

Blank HA-(E)-PLGA-rHDL $\longleftarrow$ Blank HA-(C)-PLGA-rHDL

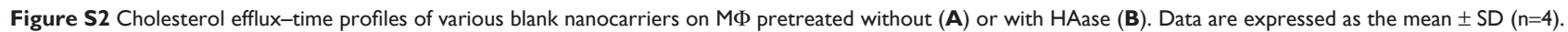
Abbreviations: MФ, RAW264.7 macrophage cell line; HAase, hyaluronidase; SD, standard deviation; PLN, PLGA-lipid nanoparticle; s-rHDL, spherical rHDL; PLGA, poly (lactic-co-glycolic acid); rHDL, reconstituted high-density lipoprotein; HA, hyaluronan; (E), electrostatically attached; (C), covalently attached.

\section{Publish your work in this journal}

The International Journal of Nanomedicine is an international, peerreviewed journal focusing on the application of nanotechnology in diagnostics, therapeutics, and drug delivery systems throughout the biomedical field. This journal is indexed on PubMed Central, MedLine, CAS, SciSearch ${ }^{\circledR}$, Current Contents ${ }^{\circledR} /$ Clinical Medicine,
Journal Citation Reports/Science Edition, EMBase, Scopus and the Elsevier Bibliographic databases. The manuscript management system is completely online and includes a very quick and fair peer-review system, which is all easy to use. Visit http://www.dovepress.com/ testimonials.php to read real quotes from published authors. 